\title{
Paediatric brainstem: A comprehensive review of pathologies on MR imaging
}

\author{
Chandan Kakkar $^{1}$ - Shruti Kakkar ${ }^{2}$ - Kavita Saggar ${ }^{1}$ - Jatinder S. Goraya ${ }^{3}$. \\ Archana Ahluwalia ${ }^{1}$. Ankur Arora ${ }^{4}$
}

Received: 18 November 2015 /Revised: 13 March 2016/Accepted: 22 April 2016/Published online: 23 May 2016

(C) The Author(s) 2016. This article is published with open access at Springerlink.com

\begin{abstract}
The brainstem is a midline structure formed by the midbrain, pons and medulla and is a home for various vital neurological centres of the human body. A diverse spectrum of disease entities can involve the brainstem, which includes infections, metabolic disorders, demyelination, vascular conditions, neurodegenerative disorders and tumours. Brainstem involvement can be primary or secondary, i.e., as part of systemic disorders. Due to the overlapping clinical presentation and symptomatology, imaging plays a decisive role in the detection, localisation and characterisation of brainstem pathologies. Magnetic resonance imaging (MRI) is the modality of choice and the use of advanced MR techniques such as diffusion-weighted imaging and spectroscopy can be especially helpful in providing a tenable diagnoses. This article is a compilation of the MR imaging manifestations of a spectrum of common and uncommon brainstem pathologies that can be encountered in the paediatric age group.
\end{abstract}

Previously presented at RSNA 2014, Chicago, IL, as an Educational Exhibit.

\section{Chandan Kakkar}

chandankakkar@yahoo.co.in

1 Department of Radiodiagnosis and Imaging, Dayanand Medical College and Hospital, Ludhiana, India

2 Division of Pediatric Haemato-oncology, Department of Pediatrics, Dayanand Medical College and Hospital, Ludhiana, India

3 Division of Pediatric Neurology, Department of Pediatrics, Dayanand Medical College and Hospital, Ludhiana, India

4 Worthing Hospital, Western Sussex NHS Foundation Trust, Lyndhurst Road, Worthing BN112DH, UK

\section{Teaching Points}

- The paediatric brainstem can be afflicted by many pathologies that may overlap clinico-radiologically.

- MRI is the best modality for the localisation and diagnosis of brainstem pathologies.

- Diffusion-weighted imaging is useful in the diagnosis of vascular and metabolic disorders.

- Occasionally, demyelination and neoplasms can be indistinguishable on imaging.

Keywords Brainstem · MRI - Demyelination - Encephalitis · Glioma

\section{Introduction}

Pathologies involving the paediatric brainstem have always been a diagnostic dilemma for paediatric neurologists as well as radiologists. Typically, the presence of long tract signs and cranial nerve palsies favour brainstem involvement; however, brainstem pathologies can have a varied presentation. Longstanding disorders can manifest with Parinaud syndrome, which suggests involvement of the dorsal midbrain, whilst the presence of internuclear ophthalmoplegia connotes involvement of the dorsomedial pons or tegmentum. Diagnosing brainstem pathologies solely on clinical considerations is not always feasible, for example, patients with acute vascular events or encephalitis typically present with altered sensorium or in a comatose state which make brainstem localisation virtually impossible. Imaging thus comes to play a paramount role in the detection and localisation of the abnormalities to the brainstem. It is however vital to understand that on imaging there can be a considerable overlap of radiological findings, with differential diagnoses ranging from infections, demyelination, to high-grade 
Table 1 Diseases of the paediatric brainstem

Diseases of the paediatric brainstem

\begin{tabular}{l} 
Vascular: \\
Infarction \\
Haemorrhage \\
Hypoxia \\
Vasculitis \\
Vascular malformations \\
Demyelination: \\
ADEM \\
Multiple sclerosis \\
Neuromyelitis optica \\
Osmotic demyelination \\
Metabolic and neurodegenerative: \\
Leigh's disease \\
Maple syrup urine disorder \\
Glutaric aciduria \\
Wilson's disease \\
Encephalitis: \\
Viral \\
Tubercular \\
Fungal \\
Parasitic \\
Tumours: \\
Tentine glioma glioma \\
Medullary glioma \\
\hline
\end{tabular}

brainstem gliomas. The histopathological diagnosis may not always be possible given the deep-seated nature of the lesions and associated morbidity that could result subsequently. Thus to achieve a plausible diagnosis imaging examinations need to be interpreted in the light of clinical details and laboratory parameters [1-3].

In this review, we present the clinico-radiological features of different brainstem pathologies in the paediatric population and discuss a comprehensive diagnostic approach to these myriad conditions. These disorders can be broadly classified into vascular pathologies, demyelination, metabolic, toxic and neurodegenerative, encephalitis and tumours (Table 1).

\section{Vascular Disorders of the Brainstem}

\section{Stroke}

Arterial ischaemic stroke remains the prime cause of cerebrovascular accidents in the paediatric age group. Some of the most important risk factors for paediatric stroke include cardioembolic phenomenon, haematological disorders such as sickle cell anaemia, infections, vasculitis, dissection and drug toxicity (L-asparaginase). Acute brainstem stroke in this age group typically presents with focal neurological deficits, hemiplegia, bulbar symptoms and rarely as locked-in syndrome. Thromboembolic infarcts usually manifest as a focal area of hyperintensity on T2-weighted and fluid attenuation inversion recovery (FLAIR) sequences in the involved region with diffusion restriction in acute stages (Fig. 1). Haemorrhagic strokes are generally sequelae of underlying vascular malformations or haematological disorders [4].

\section{Hypoxic Ischaemic Injury}

Profound hypoxia can involve the brainstem in the neonatal age group. Whilst the typical sites of involvement
Fig. 1 An 8-year-old patient with known acute leukaemia on induction therapy presented with bradycardia and hypotension: a-b Axial T2- and diffusion-weighted images reveal focal hyperintensity (arrow) showing diffusion restriction consistent with an acute infarct
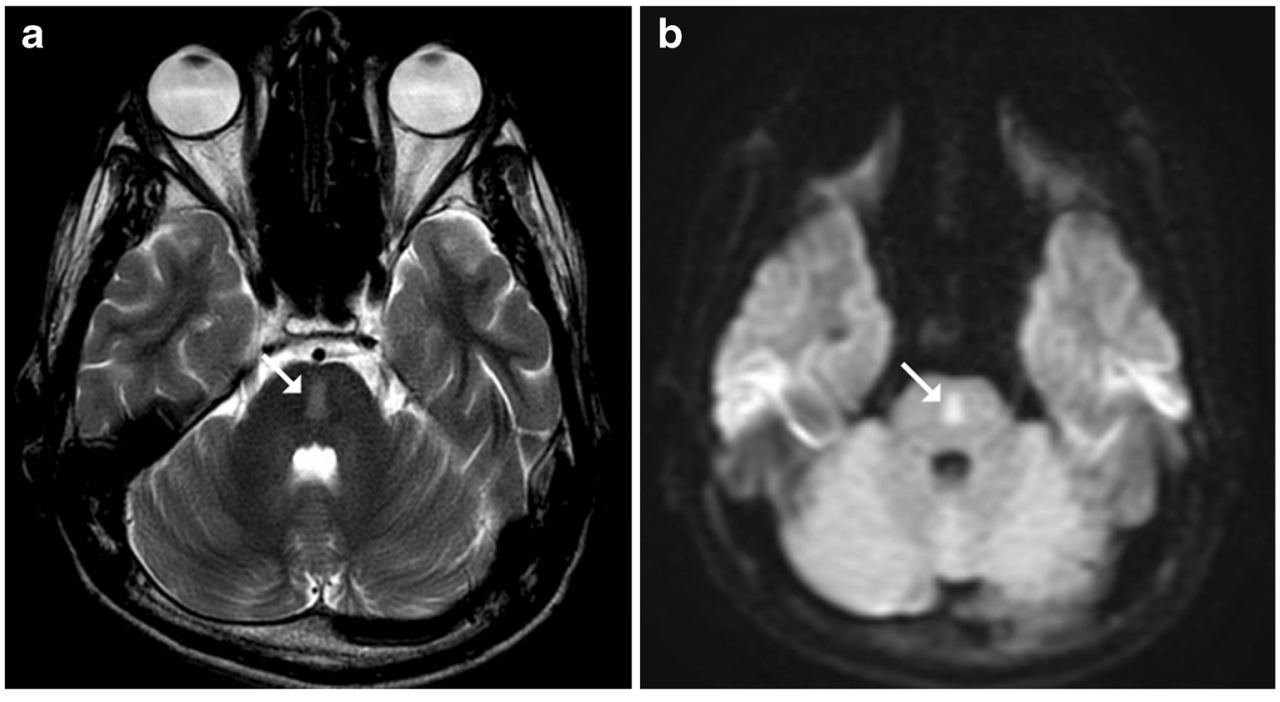

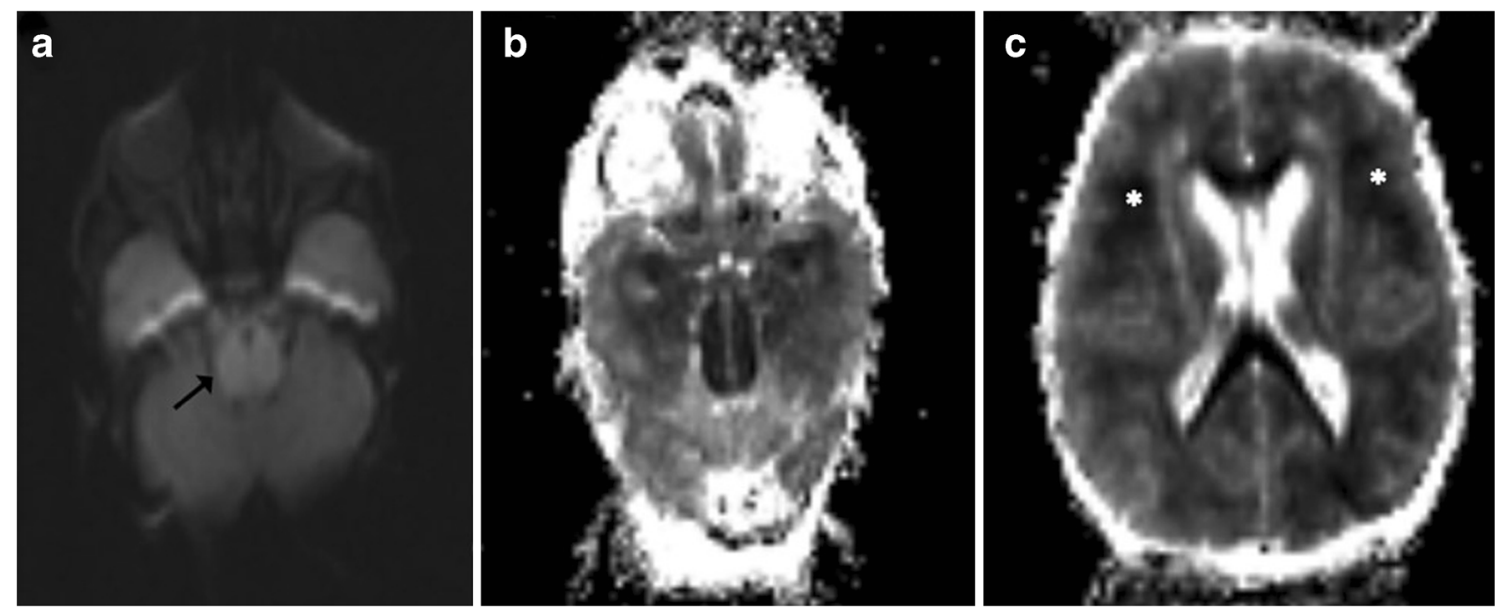

Fig. 2 Neonate on the 5th day of life with a history of obstructed labour resulting in severe hypoxia: a-b Diffusion-weighted image shows hyperintense signal involving the pons (arrow) with corresponding

include the ventrolateral thalami and corticospinal tracts in the posterior limbs of the internal capsules, midbrain and the dorsal brainstem may also get involved. Diffusionweighted imaging has the highest sensitivity in detecting early ischaemic necrosis, which manifests as areas of diffusion restriction (Fig. 2). After an initial progression the apparent diffusion coefficient (ADC) tends to normalise at 7-10 days. T1-weighted images can be especially helpful as they depict increased signal intensity within the ventrolateral thalami, posterolateral putamen, peri-Rolandic cortex and dorsal brainstem starting as early as the second day (Fig. 3) [5, 6].

\section{Vasculitis (Behçet’s Disease)}

Behçet's disease is a multisystemic vasculitic disorder of unknown aetiology characterised by perivascular inflammatory reactions predominantly centred on the venules and arterioles. Paediatric age group disease has an onset in the 2nd decade signal changes on ADC. $\mathbf{c}$ Extensive diffusion restriction is also seen in the cerebral white matter (asterisk) consistent with severe hypoxia

with a mean age of neurological involvement being 13 years. The typical triad includes oral ulceration, genital ulceration and ocular manifestations. Apart from gastrointestinal and cardiovascular involvement, Behçet's syndrome can involve the central nervous system (CNS) in up to $10-50 \%$ cases [7]. The brainstem is the most common site of involvement followed by the basal ganglia and thalami $[5,7]$. Brainstem lesions tend to be centred on the cerebral peduncles and the pons, and may be circular, linear, crescent-shaped or irregular in morphology. They are hyperintense on T2-weighted MR images, iso- to hypointense on T1-weighted images and exhibit variable post-contrast enhancement (Fig. 4). These may show true restriction or T2 shine through on diffusionweighted imaging [7].

\section{Vascular Malformations of the Brainstem}

Slow-flow vascular malformations include a group of angiographically occult vascular malformations which include
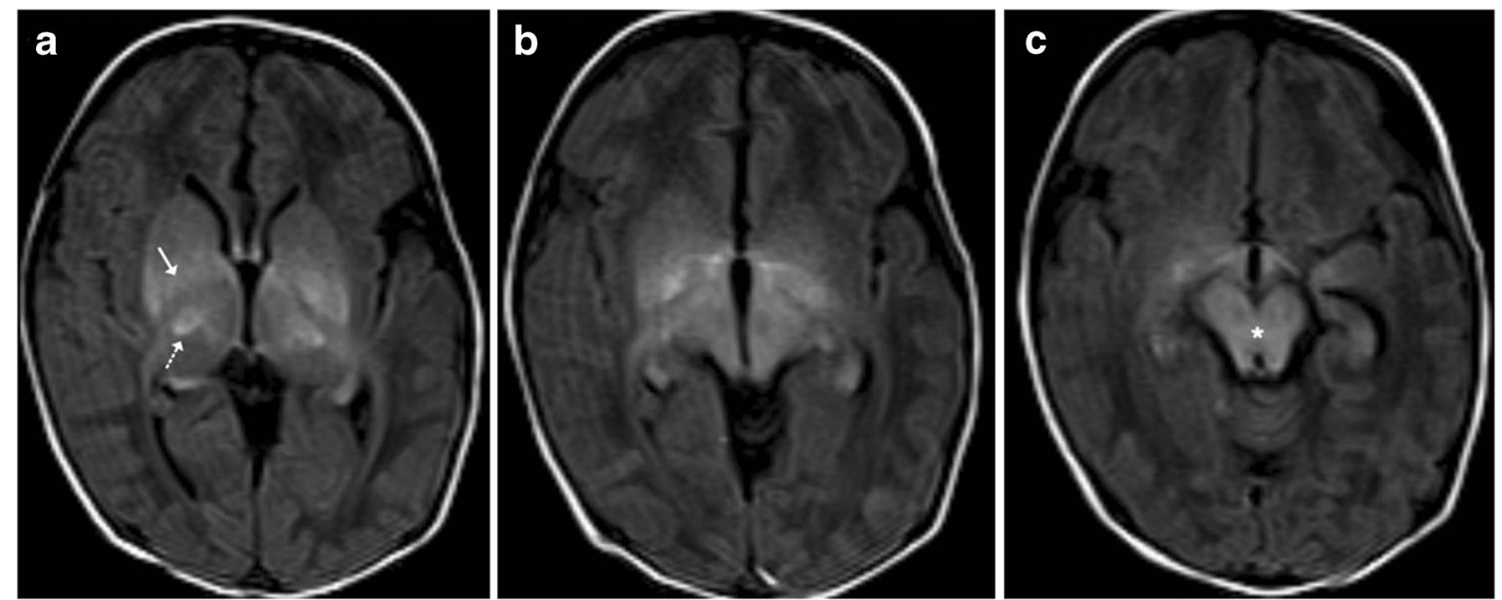

Fig. 3 Neonate with a history of delayed cry: a T1-weighted image shows symmetric increased signal intensity involving the putamina (arrow) and ventrolateral thalami (dotted arrow). b-c Similar high signal intensity is also noted involving the midbrain (asterisk) 
Fig. 4 A 16-year-old patient known to have Behçet disease: a Axial T2-weighted image shows increased signal intensity in the pons (arrow). b Mild postcontrast enhancement is noted in the involved region (arrow)

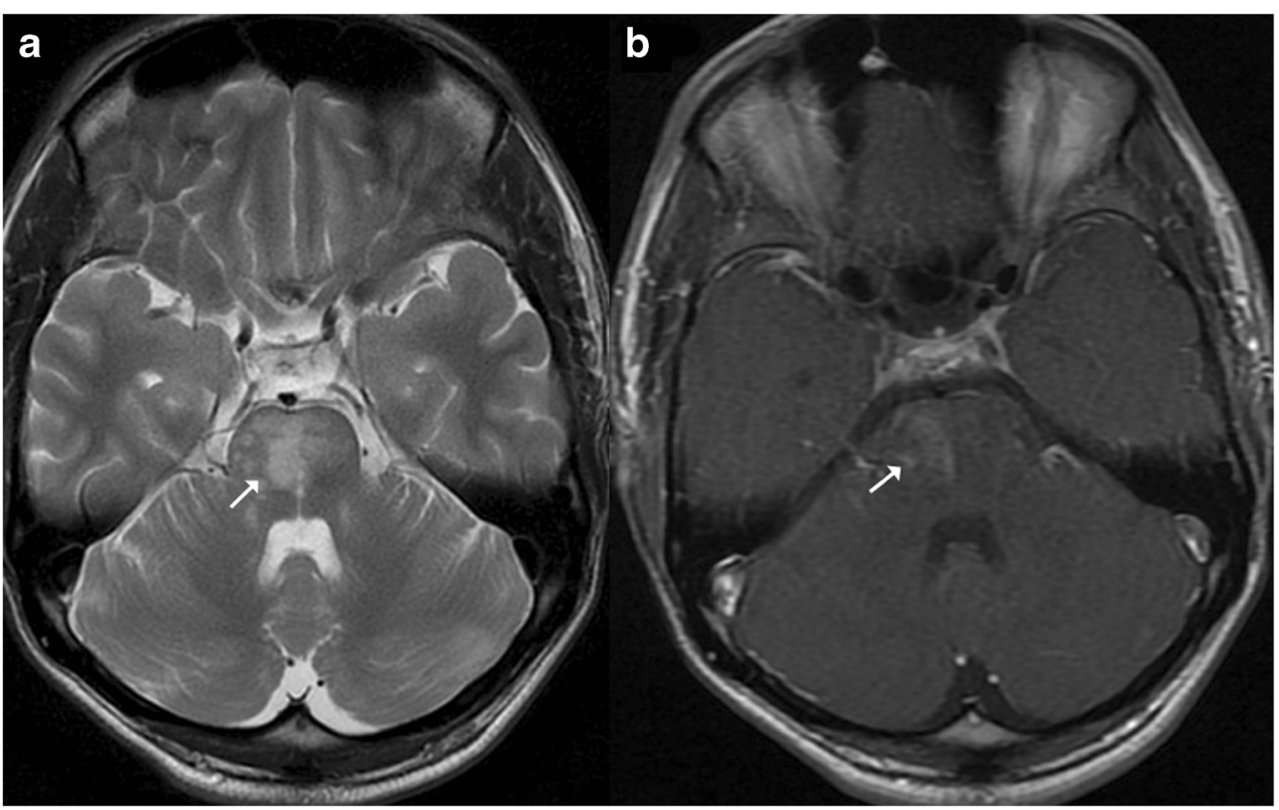

cavernous angiomas, capillary telangiectasia, developmental venous anomalies and thrombosed arteriovenous malformations. These vascular malformations can affect the brainstem and typically remain asymptomatic, being discovered incidentally on imaging. However, they can manifest neurological symptoms, for example, because of intra-lesional bleed these may present as acute neurological deficits and altered mentation, or may even present with a more indolent course with gradually worsening neurological deficits. As elsewhere, on MRI these lesions demonstrate signal characters corroborating with various stages of blood degradation. Cavernoma or cavernous haemangiomas are a cluster of thin-walled capillaries, which typically demonstrate a characteristic "popcorn" or "berry" appearance on T1- and T2-weighted images. They classically have a rim of signal loss (haemosiderin rim) on T2-weighted images and exhibit prominent blooming on susceptibility-weighted sequences (Fig. 5). Developmental venous angiomas are a venous anomaly characterised by a "caput medusa" morphology on imaging which represents a cluster of abnormally thickened veins that drain into a single larger collecting vein, which in turn drains into either a dural sinus or a deep ependymal vein (Fig. 6). Capillary telangiectasias are vascular malformations characterised by dilated capillaries interspersed with normal cerebral parenchyma. These are typically incidentally identified and may involve the brainstem (typically the pons) and spinal cord. These are often very subtle on imaging and appear as an area of blooming on gradient sequences with no or very subtle enhancement $[8,9]$.

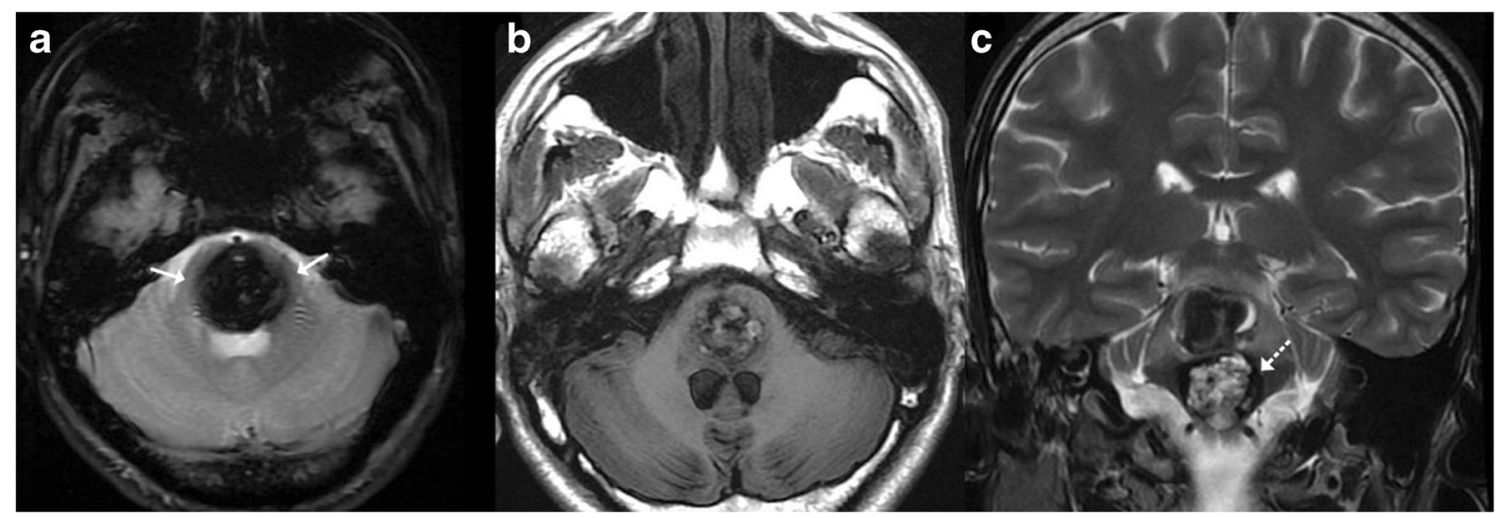

Fig. 5 A 16-year-old patient with acute onset headache: a Susceptibilityweighted image shows prominent blooming in the pons (arrow). b T1weighted images show heterogeneous areas of mixed hyper- and hypointensity consistent with blood degradation products giving a "popcorn appearance." c Heterogeneous lesion with a prominent haemosiderin rim (dotted arrow) in keeping with cavernoma. There is an associated subacute haemorrhage in the superior aspect of the lesion 


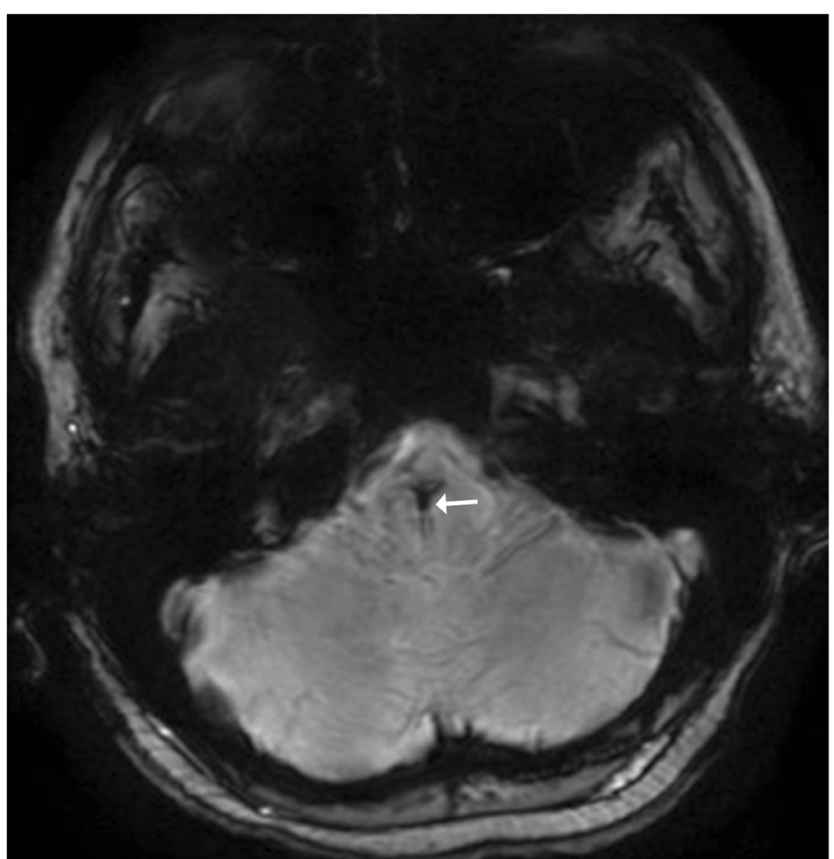

Fig. 6 A 15-year-old patient with headache and incidental finding: Susceptibility-weighted image shows "caput medusa" appearance at the pontomedullary junction consistent with a capillary telangiectasia

\section{Demyelinating Pathologies}

\section{Acute Disseminated Encephalomyelitis}

Acute disseminated encephalomyelitis (ADEM) is a monophasic, immune-mediated demyelinating CNS disorder that typically follows vaccination or viral infections. The clinical presentation is variable with no age predilection. Affected children may present with fever, headache, seizures or focal neurological deficits. MRI changes are characterised by high T2-FLAIR lesions predominantly affecting the supratentorial subcortical white matter, which tend to be asymmetric and multifocal in nature. Although the deep grey matter nuclei such as the basal ganglia and thalami may frequently become involved, brainstem involvement is quite uncommon (Fig. 7). Isolated brainstem involvement is a rare phenomenon and can be a diagnostic jigsaw both clinically and on imaging (Fig. 8). Isolated brainstem involvement has been reported as a manifestation of clinically isolated syndrome (CIS). Clinically isolated syndrome (CIS) refers to the first clinical episode of features suggestive of multiple sclerosis of which up to as many as two thirds may get converted to relapsing-remitting multiple sclerosis, although at variable speeds. Clinical response to steroids and serial MR imaging needs to be used for establishing a definite diagnosis $[1,10,11]$.

\section{Multiple Sclerosis}

Paediatric-onset multiple sclerosis constitutes between 2-5\% of all cases of multiple sclerosis. According to consensus definitions of the International Paediatric Multiple Sclerosis Study Group (IPMSSG), paediatric-onset multiple sclerosis may be diagnosed after two distinct episodes of CNS demyelination that are disseminated in time and space in individuals $<18$ years. The disease is rare before the age of 10 years and there is a female preponderance seen in children affected after the 1 st decade. The imaging findings of multiple sclerosis in the paediatric age group are not significantly different from the adult-onset disease, seen as multiple sharply demarcated white matter lesions that are hyperintense on T2-weighted images exhibiting variable degree and patterns of postcontrast enhancement (Fig. 9). The incidence of tumefactive lesions and posterior fossa plaques is higher in the paediatric age group. Brainstem plaques can cause significant expansion and simulate a neoplasm [12].
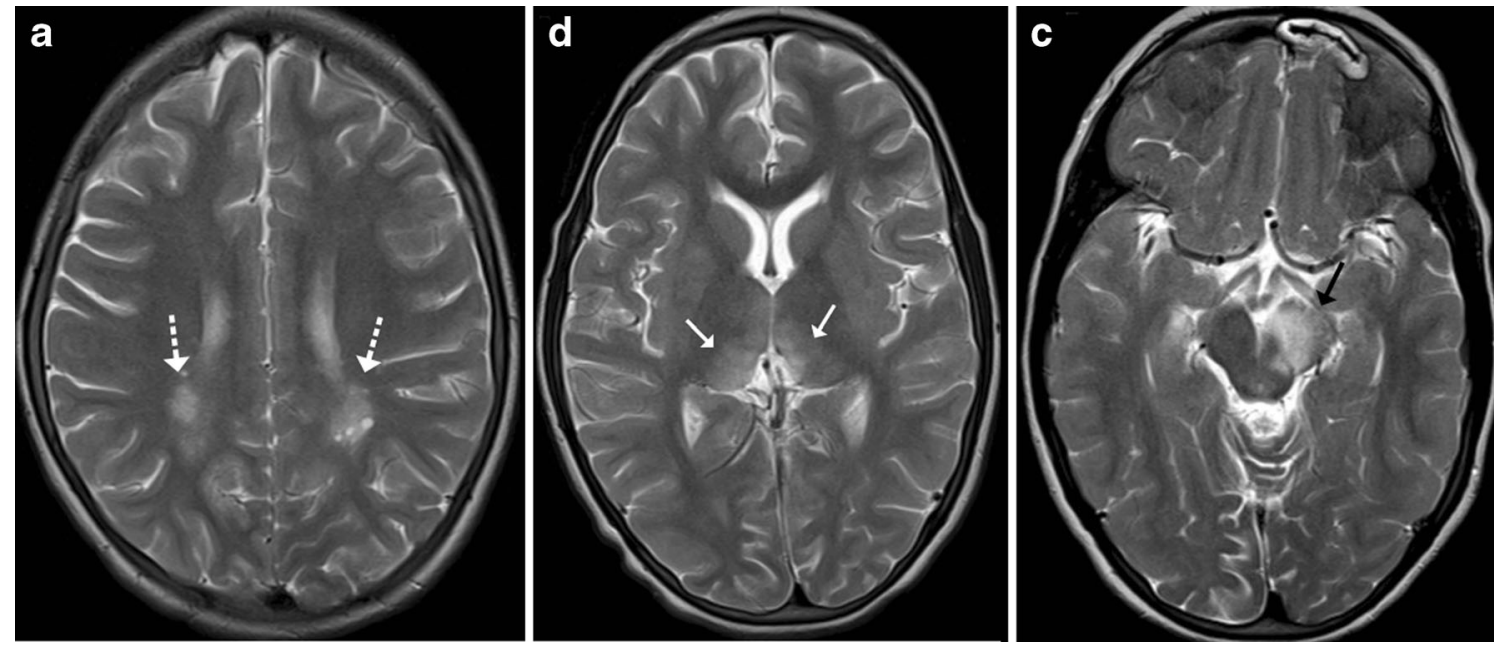

Fig. 7 A 12-year-old boy with fever and seizures: a-c Axial T2-weighted images show hyperintense signal involving the periventricular white matter, medial thalamus and left middle cerebral peduncle suggestive of demyelination 

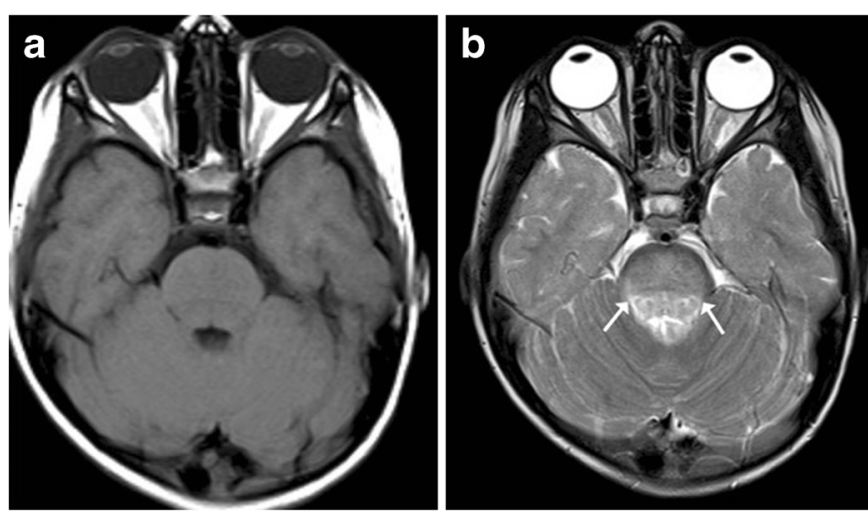

Fig. 8 Isolated brainstem ADEM in a 12-year-old patient: a-c Axial T1weighted image is unremarkable with hyperintense signal on T2 (arrows) involving the pons, which shows avid post-contrast enhancement. $\mathbf{d}$ Post
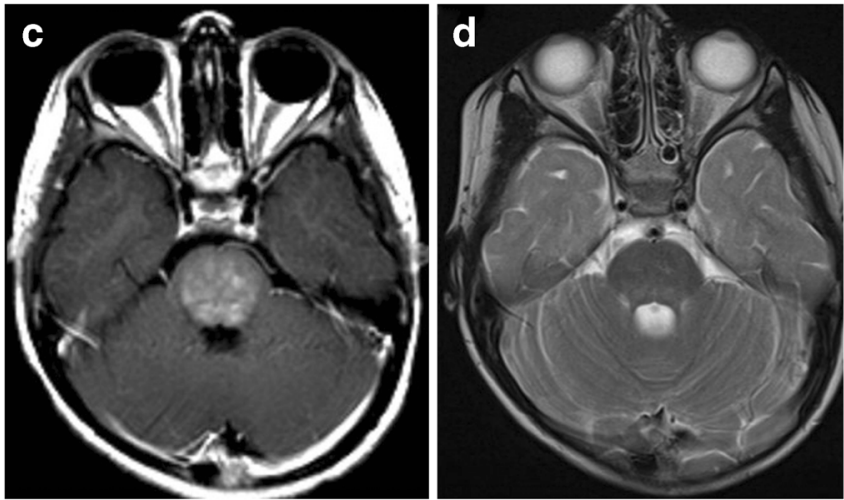

treatment: Axial T2-weighted image shows near total resolution of changes in the pons
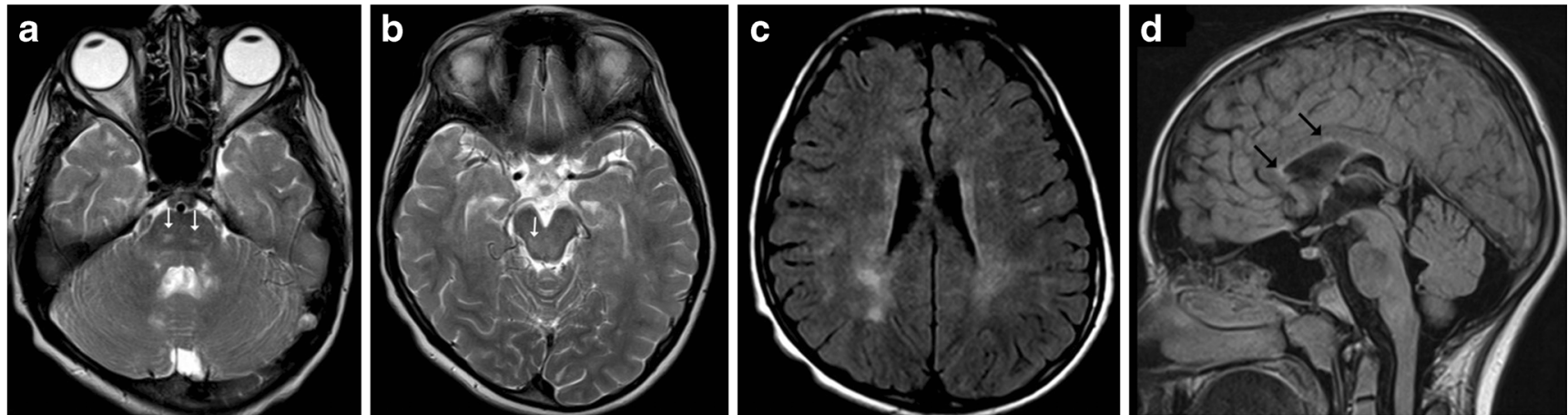

Fig. 9 A 14-year-old patient with weakness of the limbs and dysarthria: a-b Axial T2-weighted images show ill-defined increased signal intensity in the pons and midbrain (arrows). c Axial FLAIR image shows

\section{Neuromyelitis Optica (NMO)}

Neuromyelitis optica (NMO) is a form of severe inflammatory, demyelinating disease characterised by optic neuritis and periventricular white matter hyperintensity. d Sagittal FLAIR image shows increased signal in the callososeptal interface (black arrows) consistent with multiple sclerosis

longitudinally extensive myelitis, a distinct entity from multiple sclerosis. The pathogenesis involves the formation of antiaquaporin 4 antibodies, which may be seen in approximately $70 \%$ of these patients. The diagnostic criteria for NMO mandates optic neuritis and myelitis to be present with either two
Fig. 10 A 16-year-old female with neuromyelitis optica: $\mathbf{a}-\mathbf{b}$ Axial T2-weighted images show hyperintense signal involving the tegmentum (dotted arrow), periaqueductal region (arrow) of the midbrain and pons in a diffuse pattern
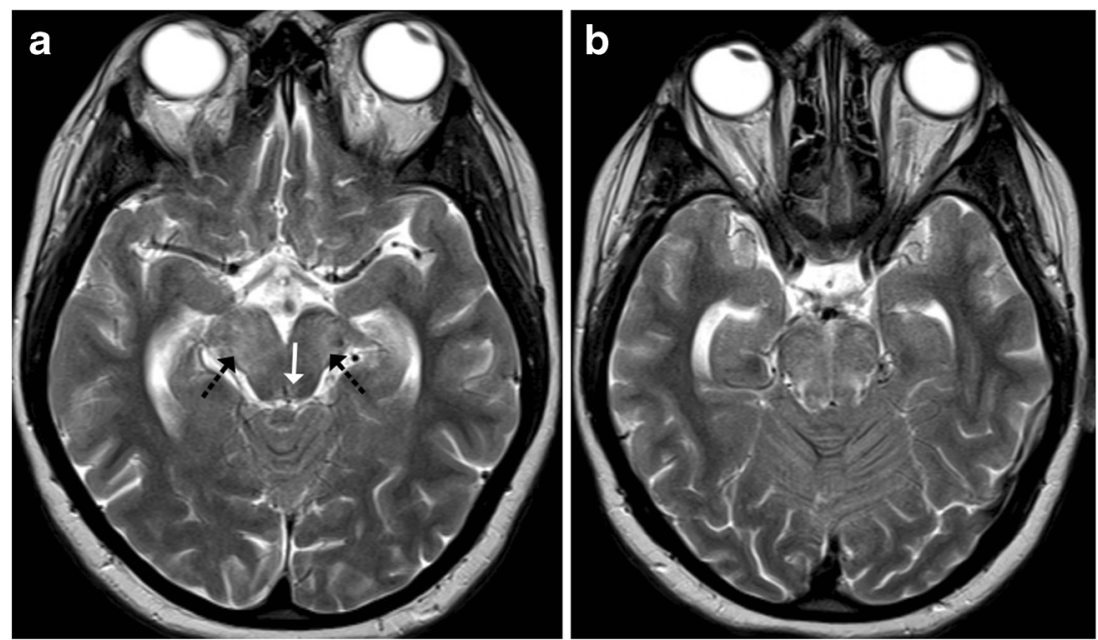

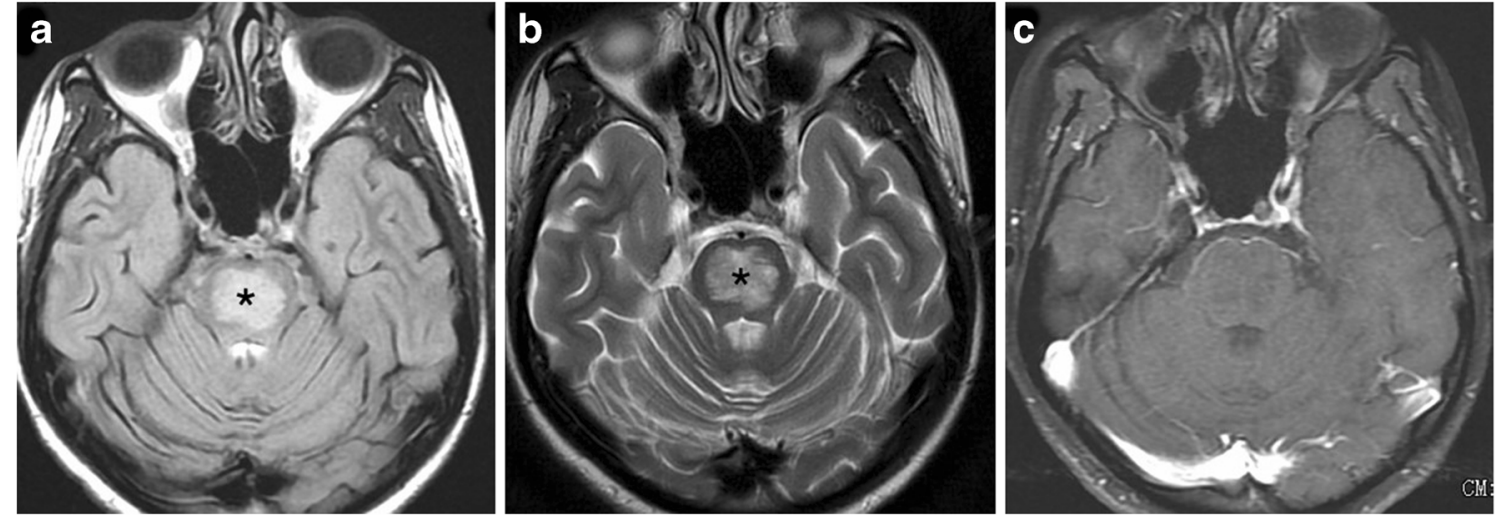

Fig. 11 A 15-year-old female with electrolyte imbalance and altered sensorium: a-b Axial FLAIR and T2-weighted images show increased signal in central pons (asterisk) with characteristic sparing of the periphery. $\mathbf{c}$ No significant post-contrast enhancement is seen

of the following criteria, i.e., contiguous spinal cord MRI lesions extending over at least three vertebral segments, onset brain MRI not meeting the diagnostic criteria of multiple sclerosis and NMO IgG seropositivity. The most common pattern of brain lesions is non-specific foci and patches of hyperintensity in subcortical and deep white matter on T2weighted or FLAIR images accompanied with characteristic sites of involvement including the periventricular regions of the third and fourth ventricles, midbrain and cerebellum (Fig. 10). High expression of anti-aquaporin antibodies in these locations is thought to be the credible explanation for the particular involvement of these sites [13].

\section{Osmotic Demyelination}

Osmotic demyelination in paediatric age groups has been reported in various disorders, which include hepatic failure, liver transplantation, chronic debilitation, malnutrition and severe electrolyte disturbances. The imaging pattern in the paediatric population is similar to the adult counterparts characterised by increased signal intensity affecting the central pons on T2-weighted images. The ventrolateral pons and corticospinal tracts are characteristically spared (Fig. 11). Occasionally, there can be involvement of the extra-pontine sites, which includes the basal ganglia, thalamus and cerebellum. The treatment of the causative factors results in reversal of the imaging findings [5].

\section{Metabolic and Neurodegenerative Diseases}

\section{Leigh Syndrome}

Leigh syndrome, also known as subacute necrotising encephalomyelopathy (SNEM), is a form of progressive mitochondrial neurodegenerative disorder of childhood typically
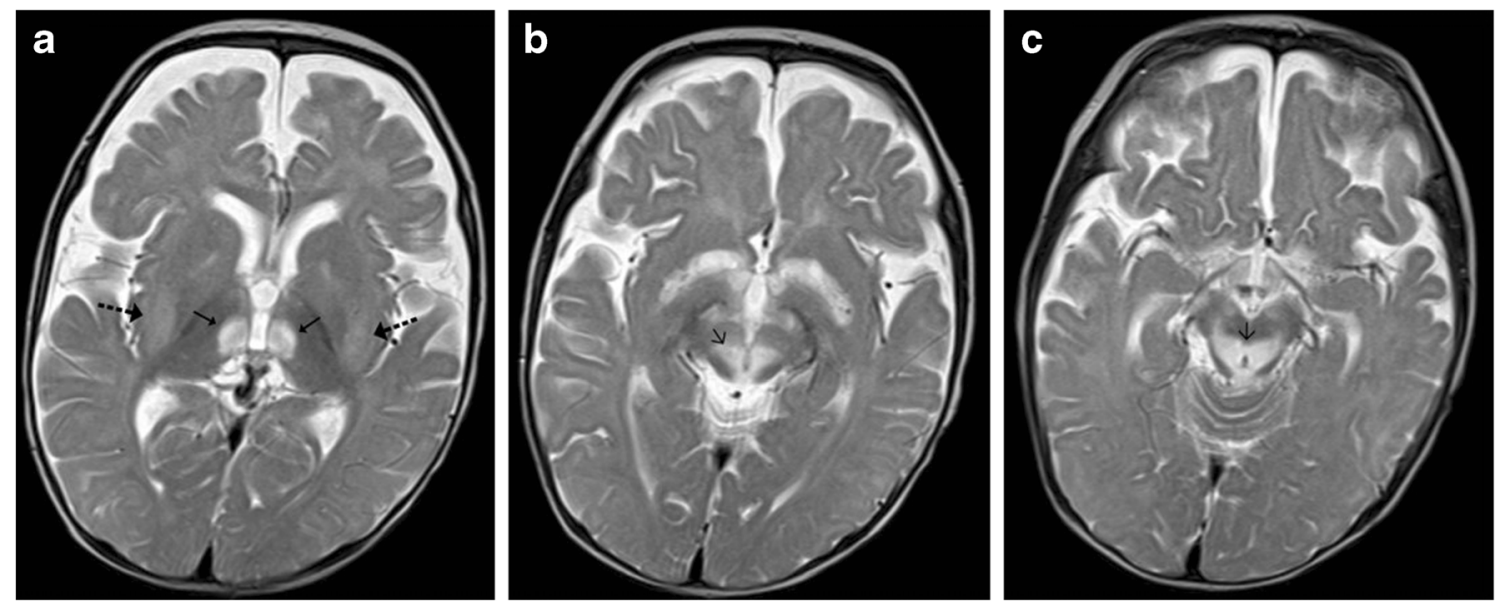

Fig. 12 A 9-month-old child with delayed milestones and seizures: a-c Axial T2-weighted images show hyperintense signal involving the putamina (dotted arrow) and medial thalamic nucleus. Caudally there is involvement of the mid brain (short arrow) with characteristic involvement of the periaqueductal region in this patient with Leigh syndrome 

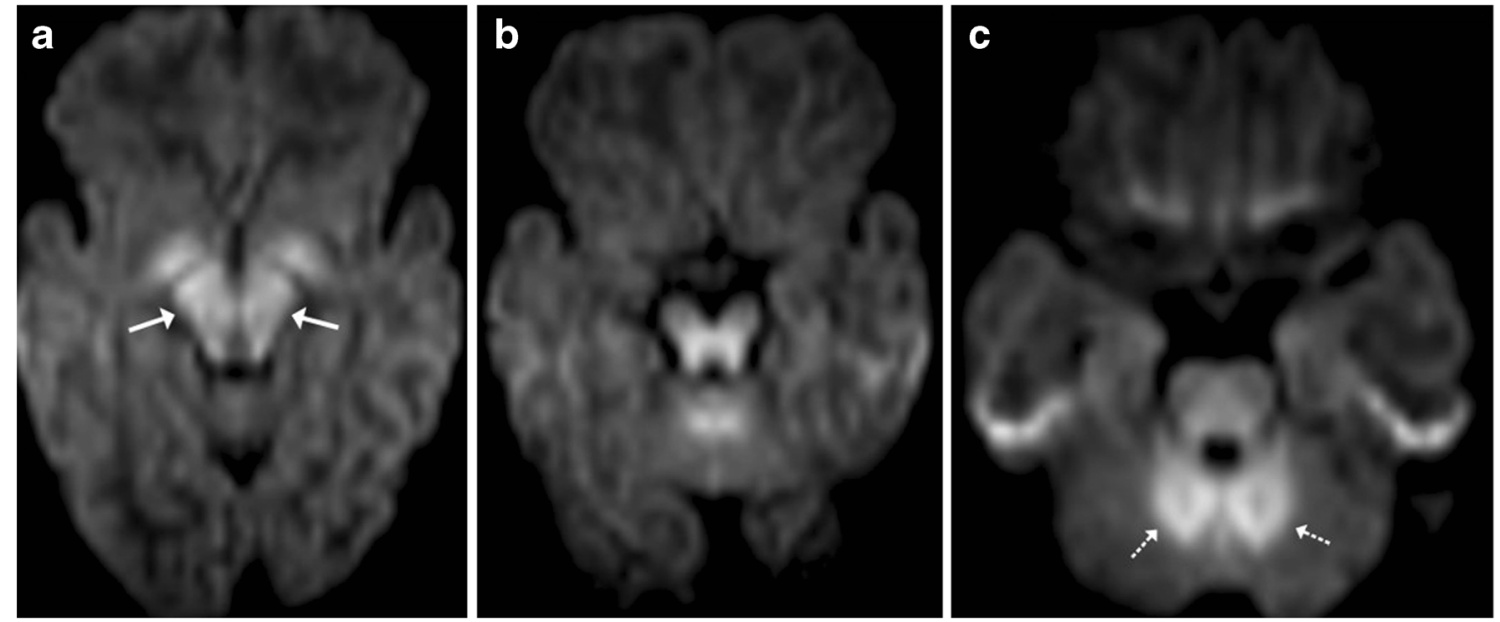

Fig. 13 Neonate with poor feeding, lethargy and ketoacidosis: a-c Axial diffusion weighted images show restricted diffusion involving the midbrain (arrow), pons and cerebellar white matter (dotted arrow) consistent with MSUD

affecting children less than 2 years old. It is invariably fatal and clinically manifests as motor disturbances with regression of milestones, ophthalmoplegia and lower cranial nerve palsies. MR imaging reveals characteristic symmetrical T2weighted high signal intensity in the periaqueductal white matter, medulla, midbrain and putamen (Fig. 12). Acute exacerbations may present with areas of diffusion restriction. CSF analysis reveals elevated lactate levels and MR spectroscopy may also reveal lactate elevation in the basal ganglia [5].

\section{Maple Syrup Urine Disease (MSUD)}

Maple syrup urine disease (MSUD) is an inborn error of amino acid metabolism that typically presents in the neonatal age group with a complex group of symptomatology including lethargy, poor feeding, seizures and a characteristic urine odour. Classically, MR imaging reveals diffuse brain swelling and diffusion restriction in myelinated brain areas, such as the posterior brainstem tracks and the central cerebellar white matter (Fig. 13). In the supratentorial compartment diffusion restriction may be seen along the posterior limbs of internal capsule, optic radiation and central corticospinal tracts [14].

\section{Wilson's Disease}

Wilson's disease in an inborn autosomal recessive error of copper metabolism that affects the liver, brain and other body tissues. Children may present in the 1st or 2 nd decades with features of liver disease, extra-pyramidal symptoms and Kayser-Fleischer (KF) rings observed on ophthalmoscopy. Elevated serum ceruloplasmin levels are helpful in confirming
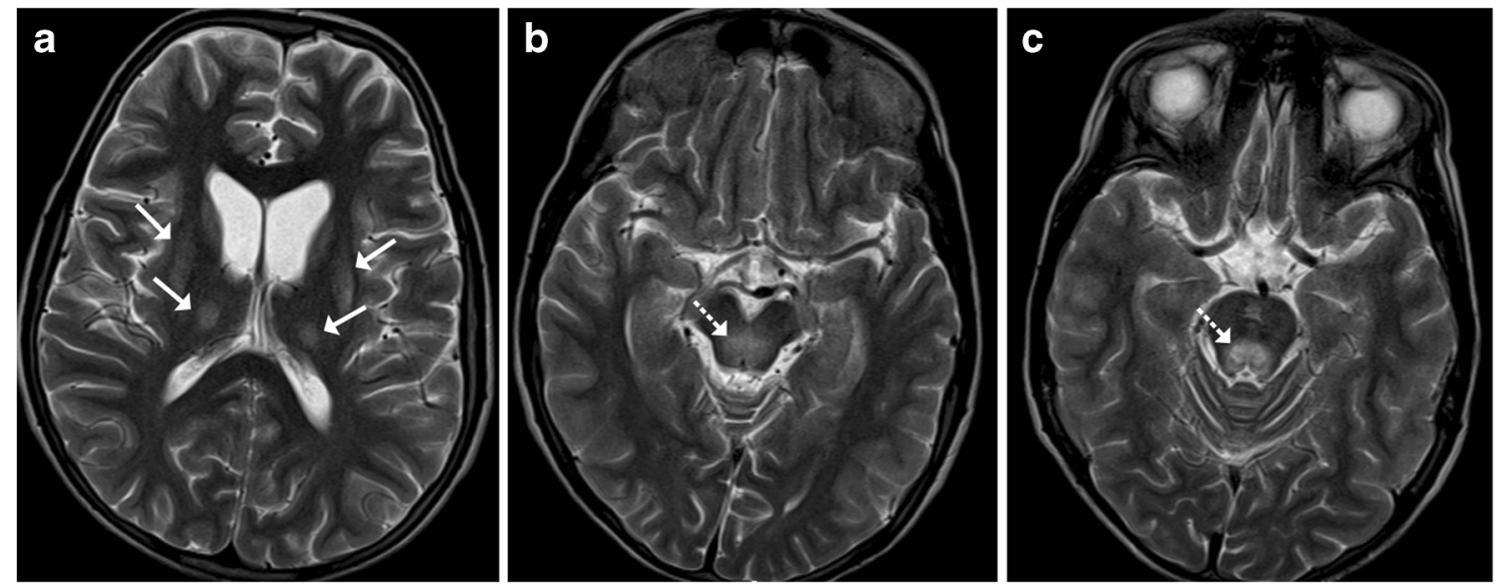

Fig. 14 A 9-year-old patient known to have Wilson's disease: a Axial T2-weighted image shows symmetrical atrophy of the basal ganglia with hyperintense signal involving the basal ganglia and thalamus (arrows). b-c Axial T2-weighted image shows increased signal intensity involving the midbrain as well as the pontine tegmentum (dotted arrows) 
Fig. 15 A 6-year-old patient with haemolytic uraemic syndrome and encephalopathy: a-b Axial T2-weighted images reveal increased signal in the basal ganglia, posterior limb of internal capsules (arrow) and midbrain (dotted arrow)

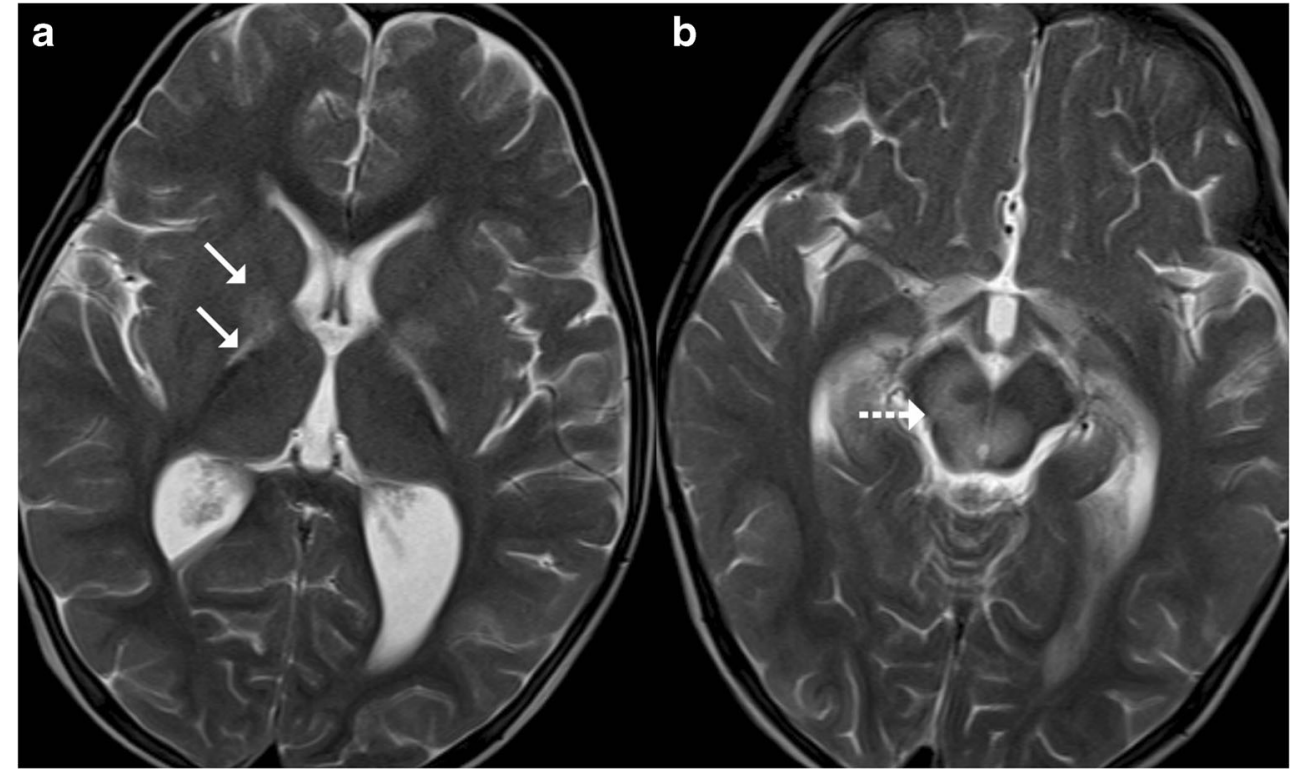

the diagnosis. Cerebral involvement typically affects the basal ganglia and thalami, which display increased T2-weighted signal intensity on MRI. The brainstem is typically involved at the midbrain and pons with $\mathrm{T} 2$ hyperintensity identified in these locations (Fig. 14). Occasionally, a "double panda sign" may be seen secondary to the signal changes affecting the midbrain tegmentum and pontine tectum $[5,15]$.

\section{Haemolytic Uraemic Syndrome (HUS)}

Haemolytic uraemic syndrome (HUS) is a complex multisystem disorder characterised by thrombocytopenia, acute renal failure and haemolytic anaemia. It is the most common cause of acute renal failure in children. CNS is the most common extra-renal site to be affected typically manifesting as altered sensorium, seizures and neurological deficits. The pathogenesis of the CNS involvement is controversial and is believed to be either toxin mediated or secondary to metabolic changes and secondary hypertension. Basal ganglia, especially dorsolateral lentiform nucleus involvement, is considered to be characteristic; arterial infarctions or white matter changes similar to posterior reversible encephalopathy can also be observed. The brainstem, cerebellum or thalami can occasionally be affected and may show foci of haemorrhage on imaging (Fig. 15) [16].

\section{Posterior reversible encephalopathy syndrome}

Posterior reversible encephalopathy syndrome (PRES) is a neurotoxic state that occurs secondary to failure of cerebral
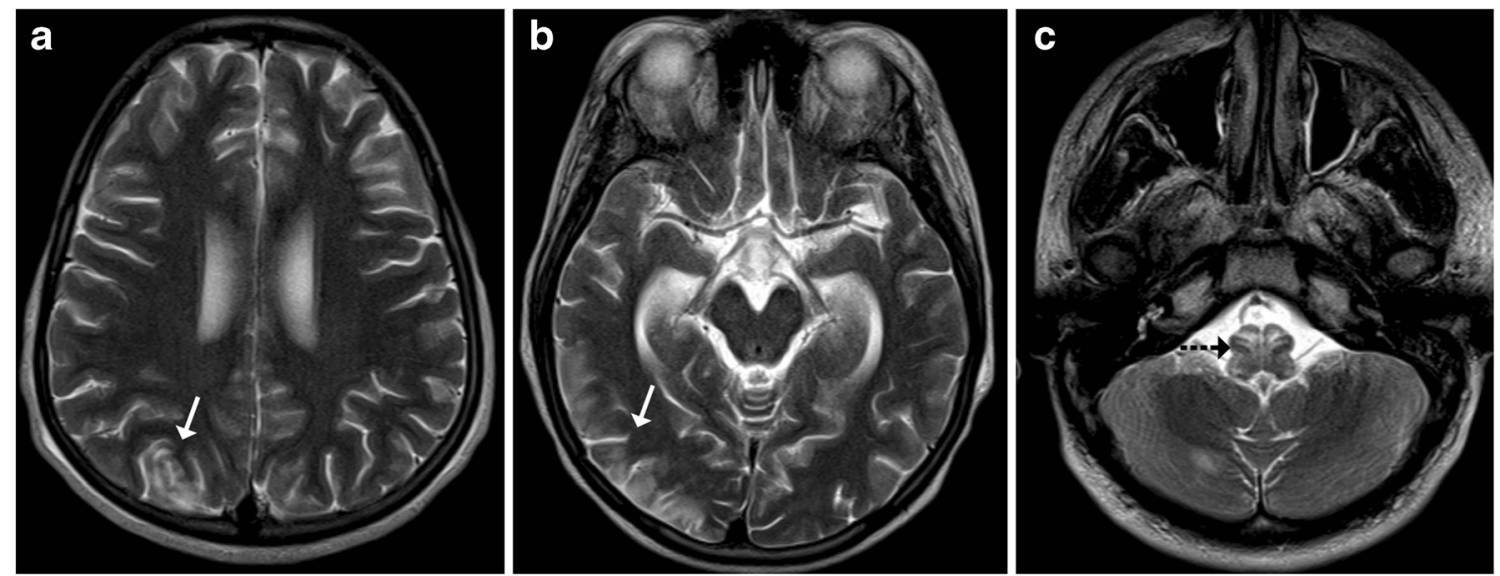

Fig. 16 A 12-year-old patient with chronic renal failure and seizures: a-c Axial T2-weighted images reveal increased signal intensity in the right parietooccipital cortex (arrow) with similar signal intensity at the cervicomedullary junction (dotted black arrow) 


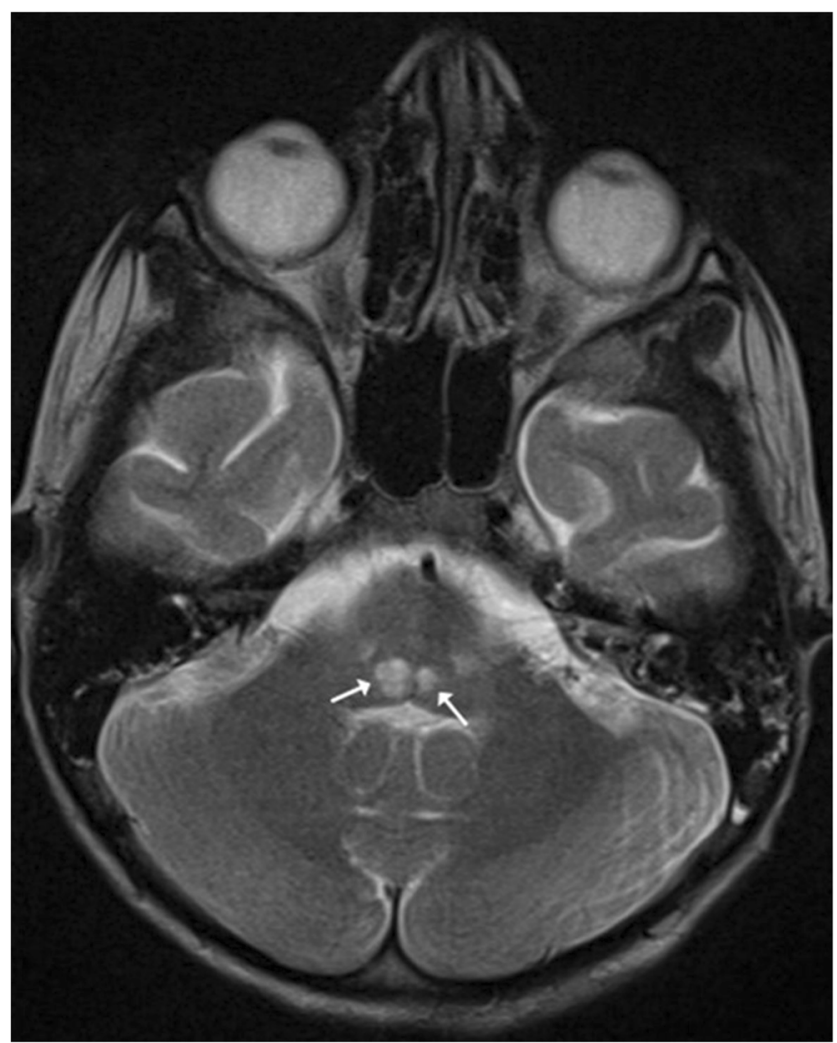

Fig. 17 A 3-year-old patient with cerebral palsy and delayed milestones: Axial T2-weighted image reveals focal tegmental hyperintensities at the level of medulla (arrows)

auto-regulation in response to acute changes in blood pressure. The disease is more commonly seen in adults; however, in the paediatric age group it can be seen in children on chemotherapy (especially in the induction phase for leukaemia), renal failure, and bone marrow or stem cell transplantation. The most common and characteristic imaging manifestation is cortical and subcortical vasogenic oedema in parieto-occipital regions. Occasionally, other parts of the cerebral hemispheres and cerebellum may be involved. The central variant is characterised by involvement of the basal ganglia and brainstem without affection of the cortical and subcortical white matter. Simultaneous involvement of the corticalsubcortical white matter and central structures may be observed sporadically (Fig. 16) [17].

\section{Central tegmental tract (CTT) lesions}

The central tegmental tract (CTT) is primarily the extrapyramidal tract connecting the red nucleus and inferior olivary nucleus. CTT lesions refer to symmetrical foci of T2 hyperintensity that involve the tegmentum and can be encountered in a variety of clinical conditions. Whilst cerebral palsy (Fig. 17) is the most common of them, CTT lesions have also been documented in congenital metabolic disorders such as non-ketotic hyperglycenemia, MSUD, mitochondrial encephalopathy, glutaric aciduria type 1 (Fig. 18), hypoxic injury and drug toxicity [18].

\section{Brainstem encephalitis}

The causes of brainstem encephalitis can be variable, ranging from infections to autoimmune aetiology. Common infectious agents responsible for brainstem encephalitis include listeria, tuberculosis, viruses, fungi and parasites.

\section{Viral Encephalitis}

Most cases of viral encephalitis have a very similar clinical and imaging manifestations and differentiation solely based
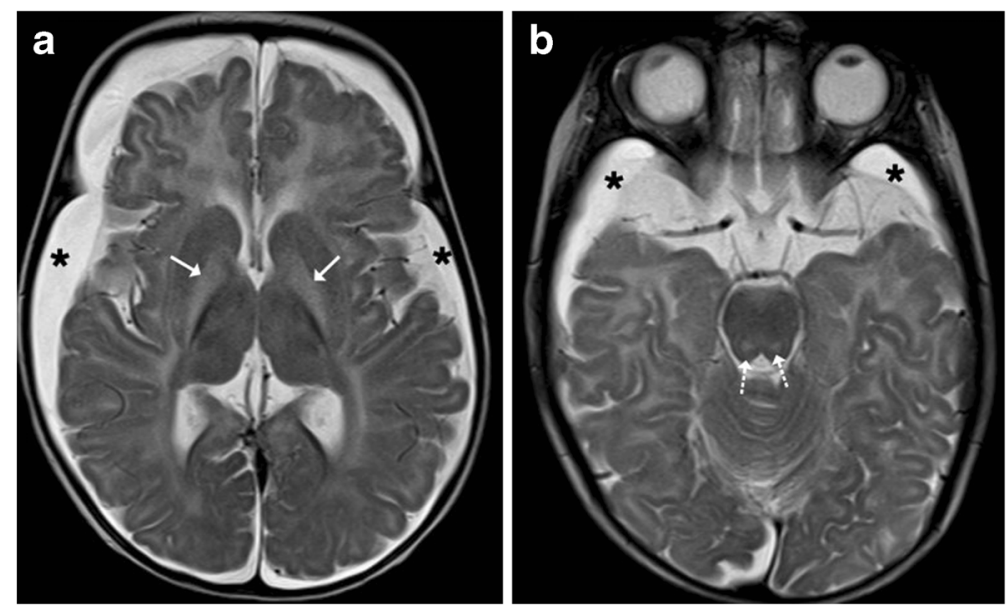

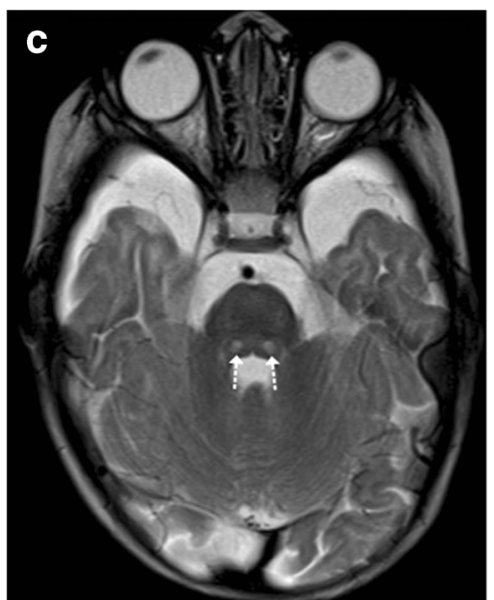

(asterisk). Prominence of the CSF spaces is noted anterior to the temporal lobe. Increased signal intensity is noted in the pontine tegmentum (dotted arrow) altered sensorium: Axial T2-weighted image shows hyperintensity involving the globus pallidi (arrow) and bilateral subdural hygromas 

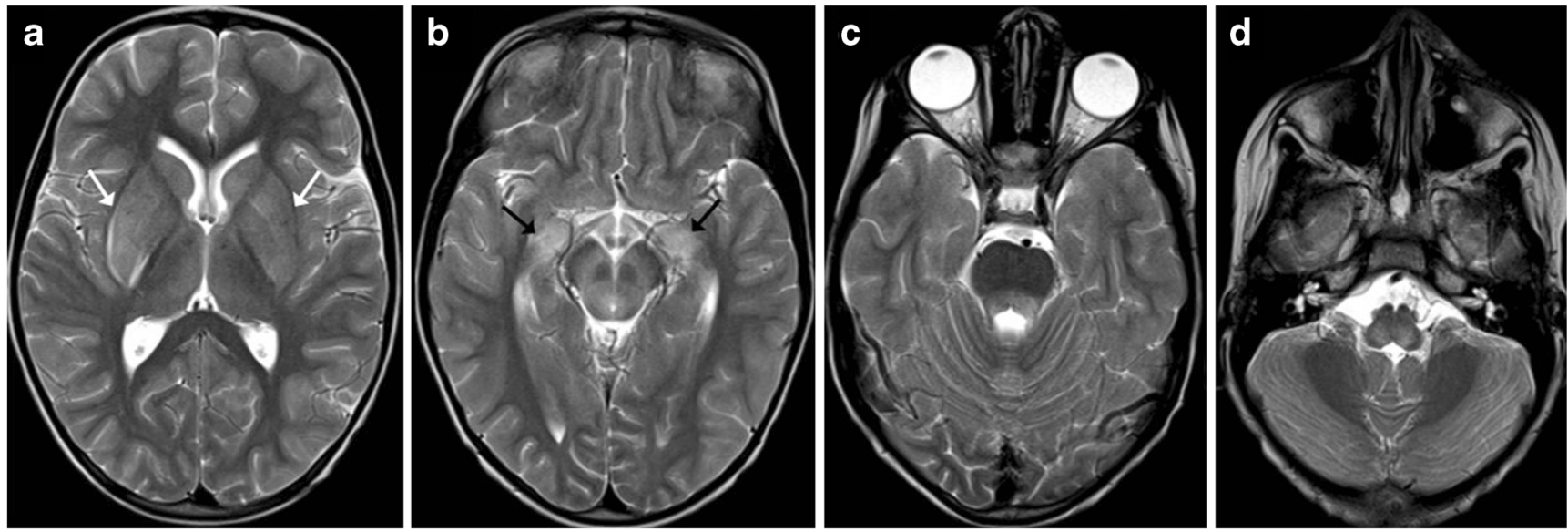

Fig. 19 A 5-year-old patient with a history of dog bite and comatose state during imaging: a-d Axial T2-weighted images reveal increased signal intensity in the basal ganglia (white arrow) with increased signal intensity

involving the medial thalami, hippocampi (black arrow), midbrain, pons and medulla

on imaging alone can be extremely challenging. Rabies encephalitis has a propensity to affect the spinal cord, brainstem, basal ganglia, thalami, hypothalamus and hippocampi (Fig. 19); needless to say, that the clinical information is paramount as a very similar involvement can be seen in other causes of viral encephalitis. Similarly, in a clinical context of suspected viral encephalitis, involvement of the brainstem with bilateral thalamic involvement can suggest Japanese encephalitis (Fig. 20). Herpes encephalitis apart from the limbic system can also involve the brainstem $[19,20]$.

\section{Tuberculosis and Fungal}

Brainstem involvement by tuberculosis can be isolated or as a part of disseminated CNS disease. Leptomeningeal involvement can be in the form of basal meningitis (Fig. 21). The signal intensity of the parenchymal lesions vary on T1-and T2- weighted sequences depending on the stage of the granuloma though most of these lesions tend to show peripheral rim enhancement following intravenous contrast administration (Fig. 22) [21, 22]. Understandably, isolated brainstem lesions can be extremely challenging as the appearances may be indistinguishable from neoplastic processes like gliomas. Fungal infections of CNS are usually opportunistic in nature occurring in immunocompromised patients thus clinical history can prompt the radiologist to consider them in appropriate settings. Intracranial involvement is either secondary to haematogenous dissemination or extension from the adjacent sinus disease. The frequent occurrence of significant parenchymal destruction is often associated with high fatality. Mucormycosis and aspergillosis owing to their angioinvasive propensity tend to result in in parenchymal haemorrhages which can be helpful in clinching the diagnosis (Fig. 23) [22].

\section{Parasitic (Cerebral Malaria)}

Cerebral manifestations in malaria occur because of sequestration of infected erythrocytes in the microcirculation
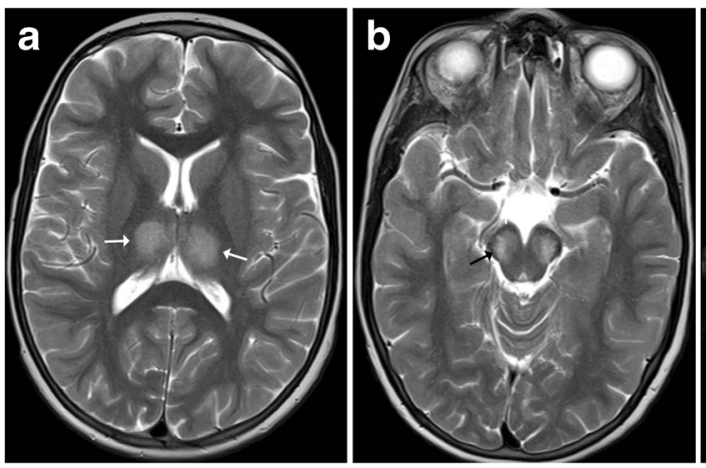

Fig. 20 A 10-year-old patient with fever and altered sensorium: a-c Axial T2-weighted images show near symmetrical increased signal intensity in the medial thalami (white arrow), middle cerebral peduncles (black arrow) and pons (asterisk). The possibility of viral encephalitis,
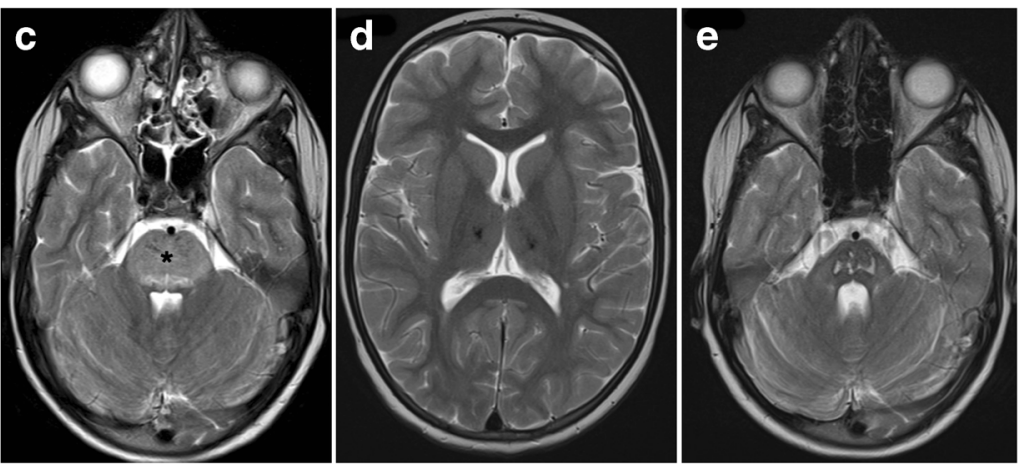

likely Japanese encephalitis, was considered. d-e Follow-up MRI: Axial T2-weighted images show hypointense foci (possibly remote haemorrhage) in the thalami with volume loss and an area of gliosis in the pons 
Fig. 21 A 6-year-old patient with fever and headache: $\mathbf{a}-\mathbf{b}$ Axial contrast enhanced $\mathrm{T} 1$ image shows marked meningeal enhancement in basal cisterns (arrow), middle cerebral artery cisterns (dotted arrows) and dilated lateral ventricles (thin arrows arrow) in a case of tubercular meningitis
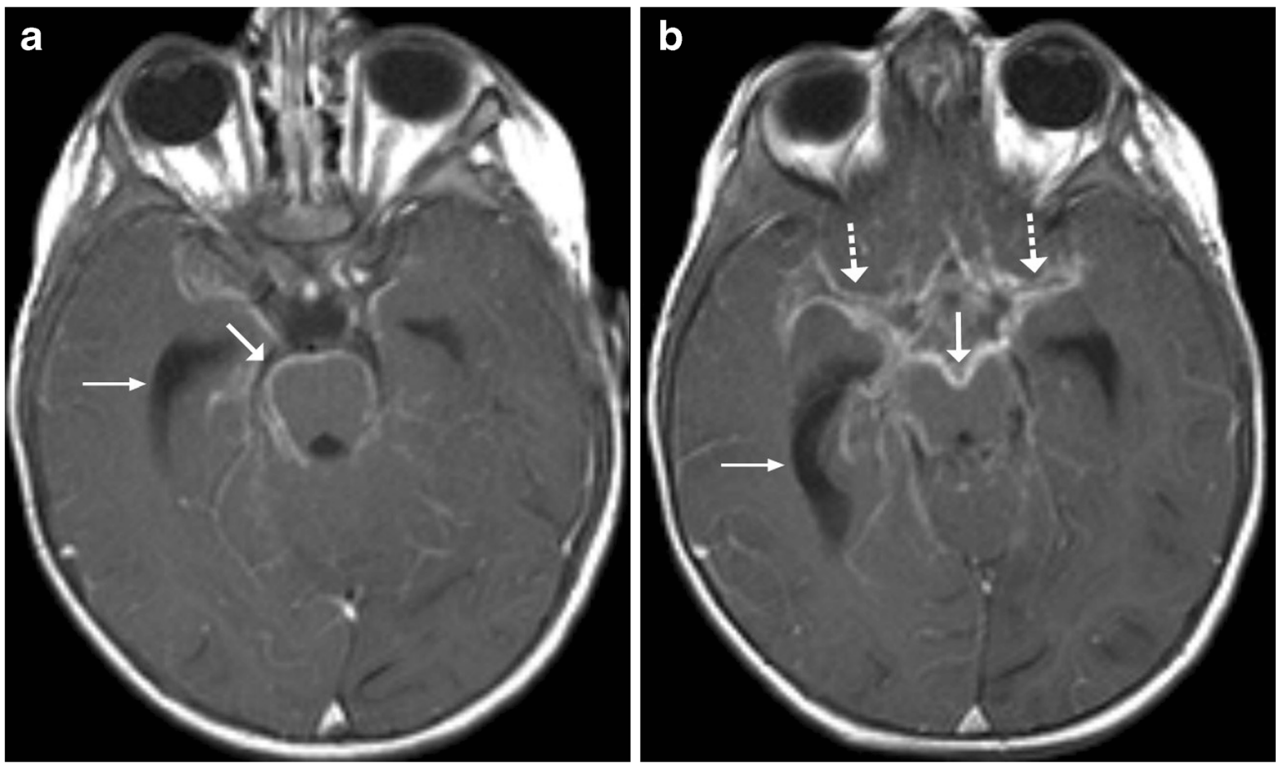
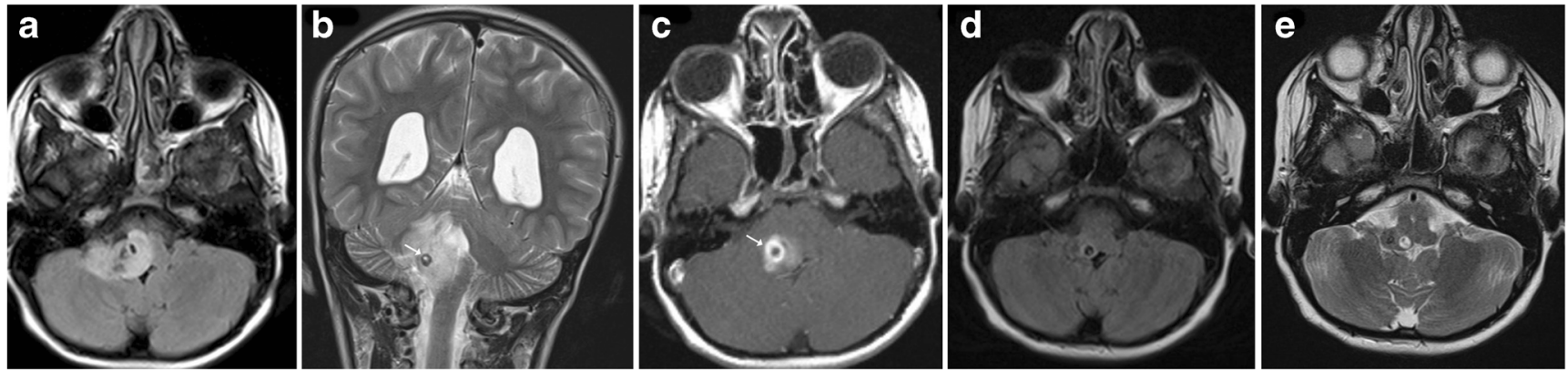

Fig. 226 year old with headache, vomiting and fever: a Axial FLAIR image reveals hyperintense signal involving the medulla with extension into the adjacent cerebellar white matter. $\mathbf{b}$ and $\mathbf{c}$ Coronal T2-weighted image reveals small lesion with peripheral hypointensity at pontomedullary junction (arrow), the lesion shows peripheral

resulting in multiple foci of ischaemic necrosis. It typically manifests as encephalopathy and coma and is frequently fatal. MRI features are highly variable and may enhancement on post contrast images. Possibility of CNS tuberculoma was considered. d-e Follow-up after 3 months on anti-tubercular treatment: Axial FLAIR and T2-weighted images show significant reduction in the hyperintensity involving the medulla with residual lesions. Clinically the child showed significant improvement

include multiple cortical infarcts with or without involvement of the deep grey matter nuclei, corpus callosum, cerebellum and brainstem. Involved areas exhibit T2-
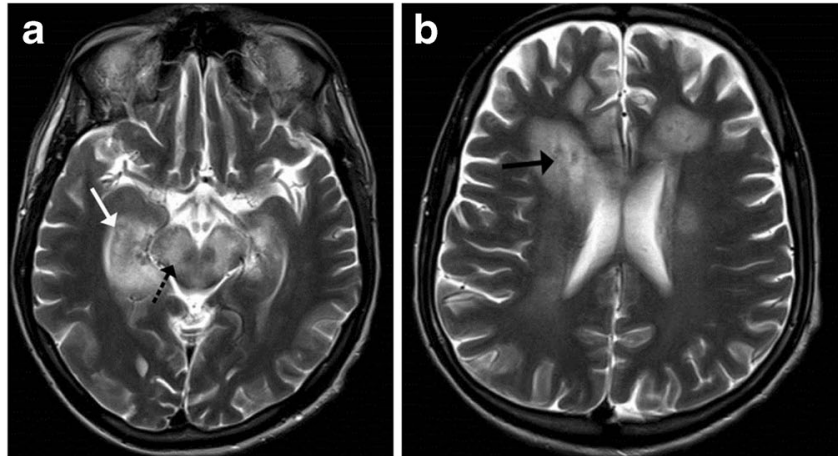

Fig. 23 A 16-year-old patient with acute promyelocytic leukaemia on chemotherapy with angioinvasive aspergillosis: a-b Axial T2- weighted MRI shows increased signal intensity in the temporal lobes (white arrow)
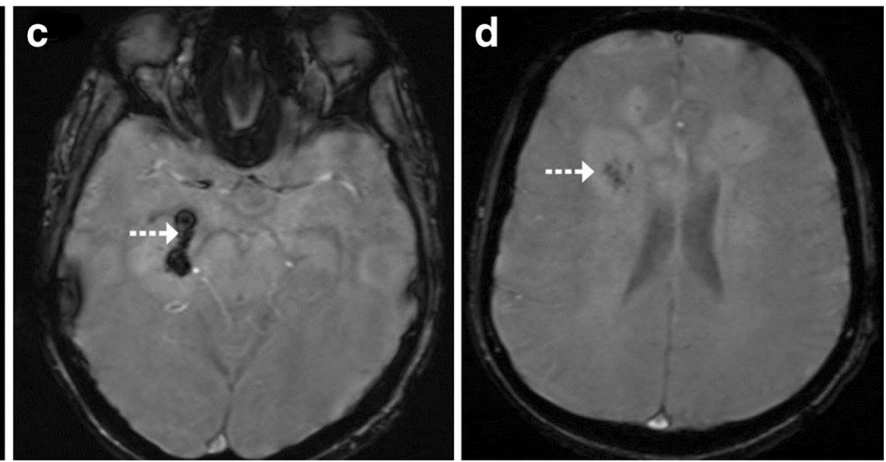

with involvement of the cerebral peduncles. Similar signal intensity is noted in the frontal white matter bilaterally. c-d Significant blooming (dotted arrows) is noted on susceptibility-weighted imaging 

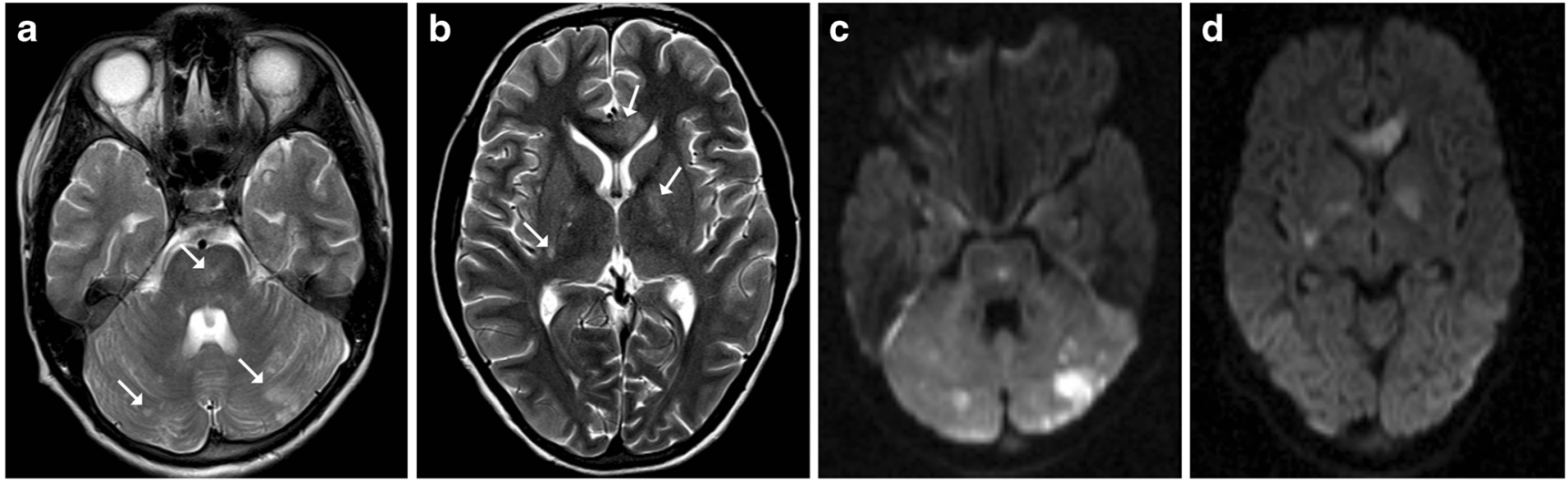

Fig. 24 A 13-year-old patient with cerebral malaria: Axial T2-weighted MRI reveals hyperintense foci showing diffusion restriction in the left cerebellum, pons, bilateral basal ganglia and corpus callosum (arrows) corresponding to areas of cytotoxic oedema
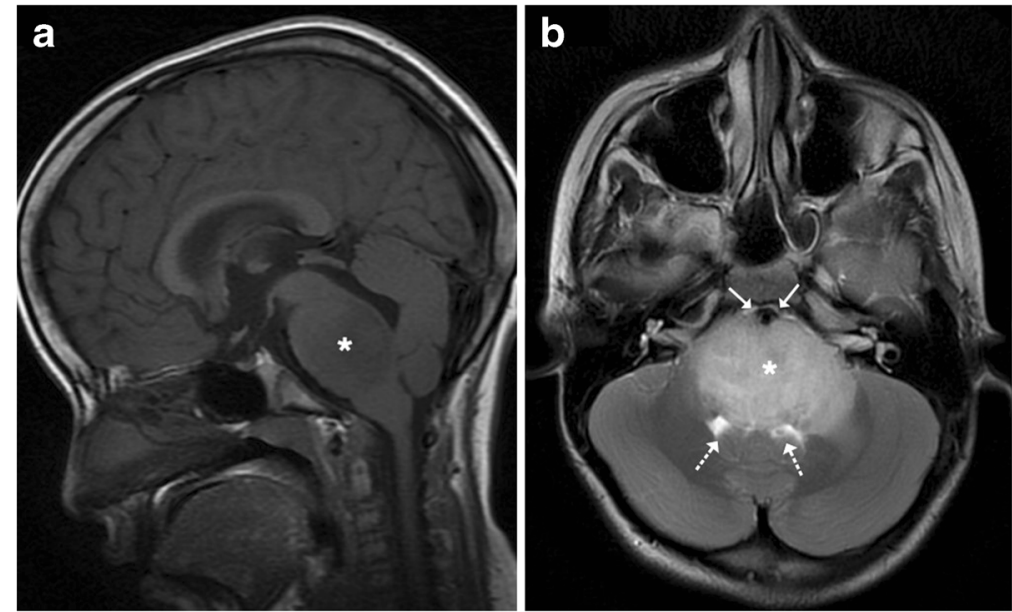

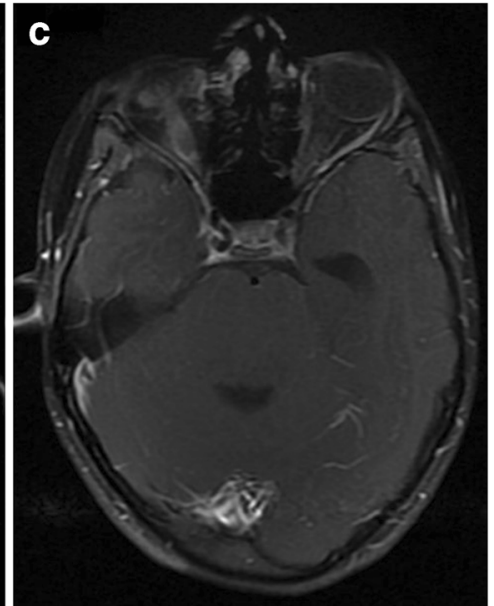

engulfed by the lesion. Posteriorly the fourth ventricle is compressed (dotted arrow) (flat fourth ventricle sign). c No significant contrast enhancement is noted within the lesion

Frequently, there are foci of haemorrhages that are best appreciated on gradient sequences (Fig. 24) [22, 23].
FLAIR hyperintensity and the presence of multifocal areas of diffusion restriction connote cytotoxic oedema. weighted image shows expansion of the pons with hypointense signal (asterisk). b Axial T2-weighted image shows increased signal intensity involving the pons (asterisk) with the basilar artery (arrow) appearing
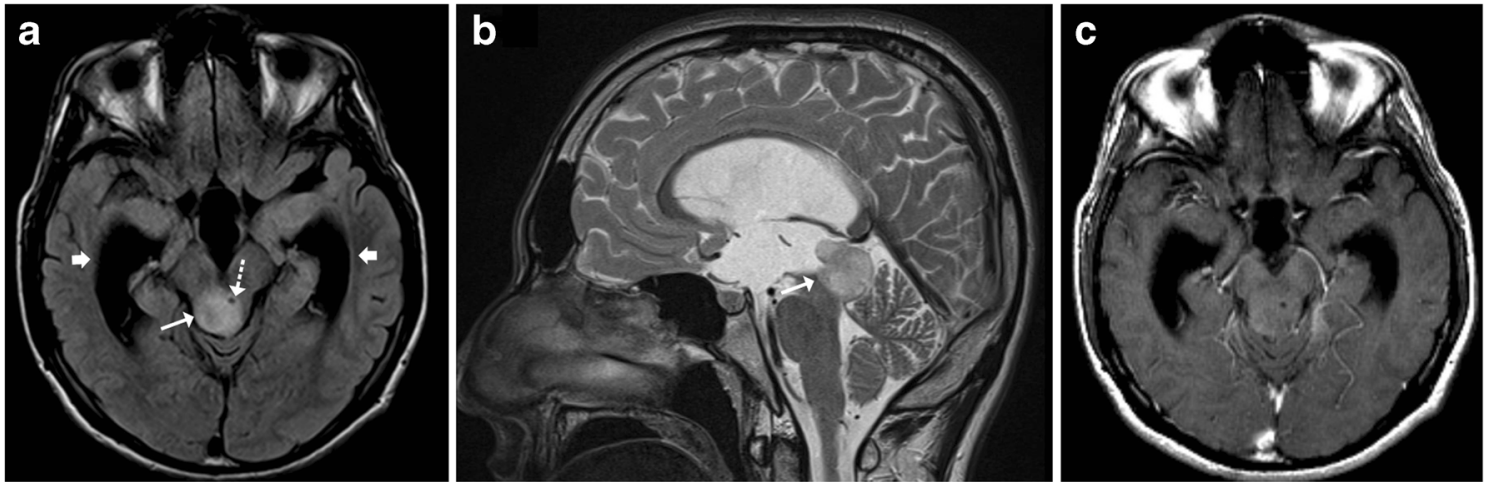

Fig. 26 A 16-year-old male with headache and vomiting: a-b Axial FLAIR and T2-weighted sagittal MRI shows a relatively well-defined hyperintense lesion involving the tectum (arrow). The aqueduct is encased and compressed (dotted arrow) with resultant dilatation of the temporal horns (block arrow). c No significant contrast enhancement is noted in keeping with a glioma 


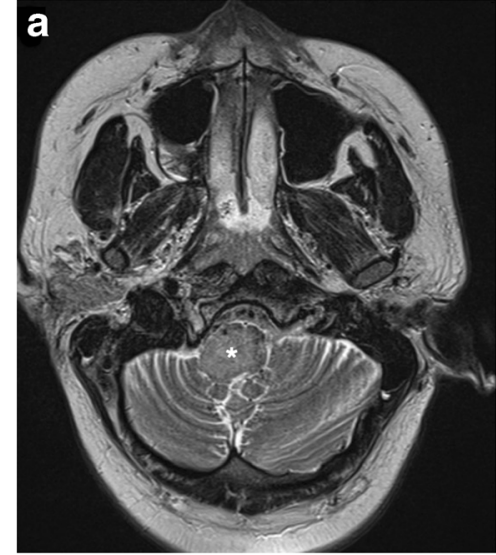

Fig. 27 A 15-year-old male with altered headache and lower cranial nerve palsies: a-b Axial and sagittal T2-weighted image reveals an illdefined hyperintense expansive lesion involving the medulla $(*)$, which is

\section{Tumours of the Brainstem}

\section{Brainstem Glioma}

Brainstem gliomas comprise a heterogeneous group of tumours having variable histological patterns and prognostic implications. These lesions comprise $10-20 \%$ of the intracranial tumours in the paediatric age group with a majority of them occurring in the first decade of life (peak incidence: $3-7$ years). The lesions are sub-classified as diffuse, focal, exophytic and cervicomedullary. Diffuse lesions tend to affect the pons and present as long tract signs; clinically, ataxia or lower cranial nerve palsies are associated with a dismal prognosis. These lesions expand the pons and cause anterior displacement of the basilar artery, which appears engulfed by the lesion. There can be flattening of the fourth ventricle resulting

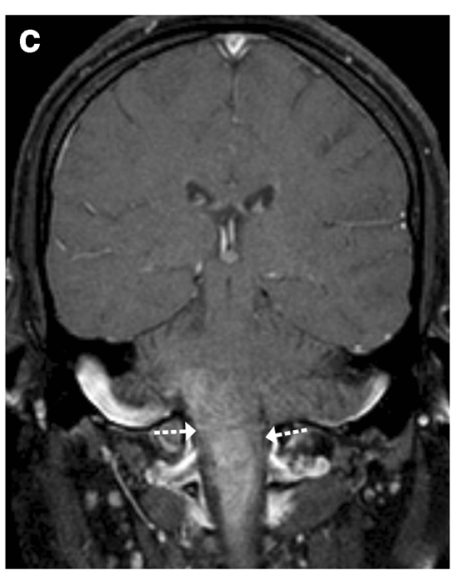

extending caudally to involve the cervical cord (arrow). c Post contrast the lesion shows homogeneous enhancement (dotted arrows)

in hydrocephalus (flat fourth ventricle sign). Most lesions are hypo- to isointense on T1-weighted images, hyperintense on T2-weighted images, and display no or minimal enhancement (Fig. 25). Tectal gliomas tend to be focal and have a better prognosis as these are low-grade astrocytomas. These lesions typically cause mild expansion of the tectal plate resulting in narrowing of the aqueduct and obstructive hydrocephalus. The lesions are isointense on T1-weighted, hyperintense on T2-weighted images and do not show post-contrast enhancement (Fig. 26). Medullary tumours have a variable morphology and may be exophytic, focal or diffuse in nature. Imaging findings are non-specific although mostly associated with the expansion of the medulla. Diffuse lesions can extend cranially to involve the pons and caudally to involve the cervical spinal cord (Fig. 27). The enhancement characteristics of these lesions are inconsistent and variable [24].
Fig. 28 A known case of neurofibromatosis type I with hydrocephalus: a-b Axial T2weighted images show mild expansion of the midbrain and pons with multiple hyperintense lesions involving the midbrain, pons and cerebellar peduncle (arrow). Periaqueductal hyperintensity with narrowing of the aqueduct (black arrow) resulting in third ventricular dilatation (dotted arrow)
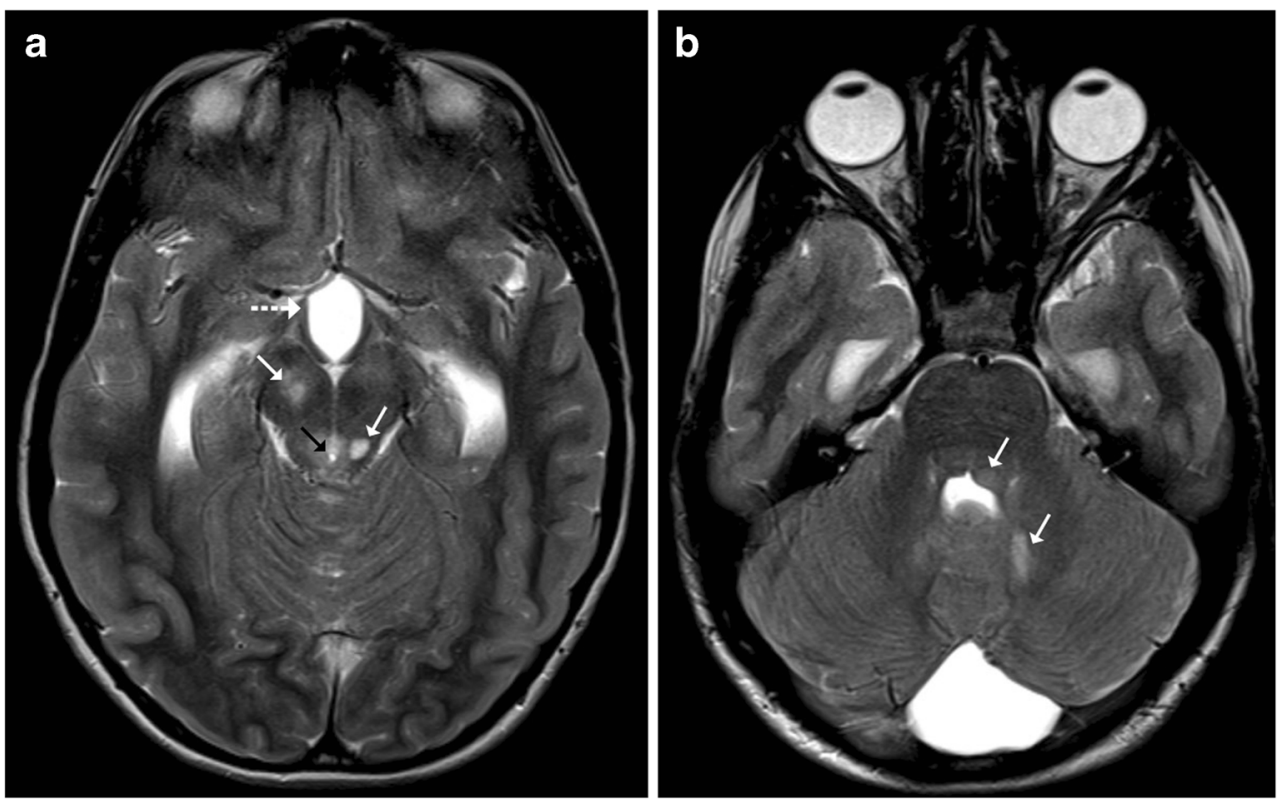
Fig. 29 A 5-year-old patient with cerebellar medulloblastoma: a-b Axial T2-weighted and diffusionweighted image shows a large hypointense mass showing restriction in the left cerebellar hemisphere causing severe brainstem compression
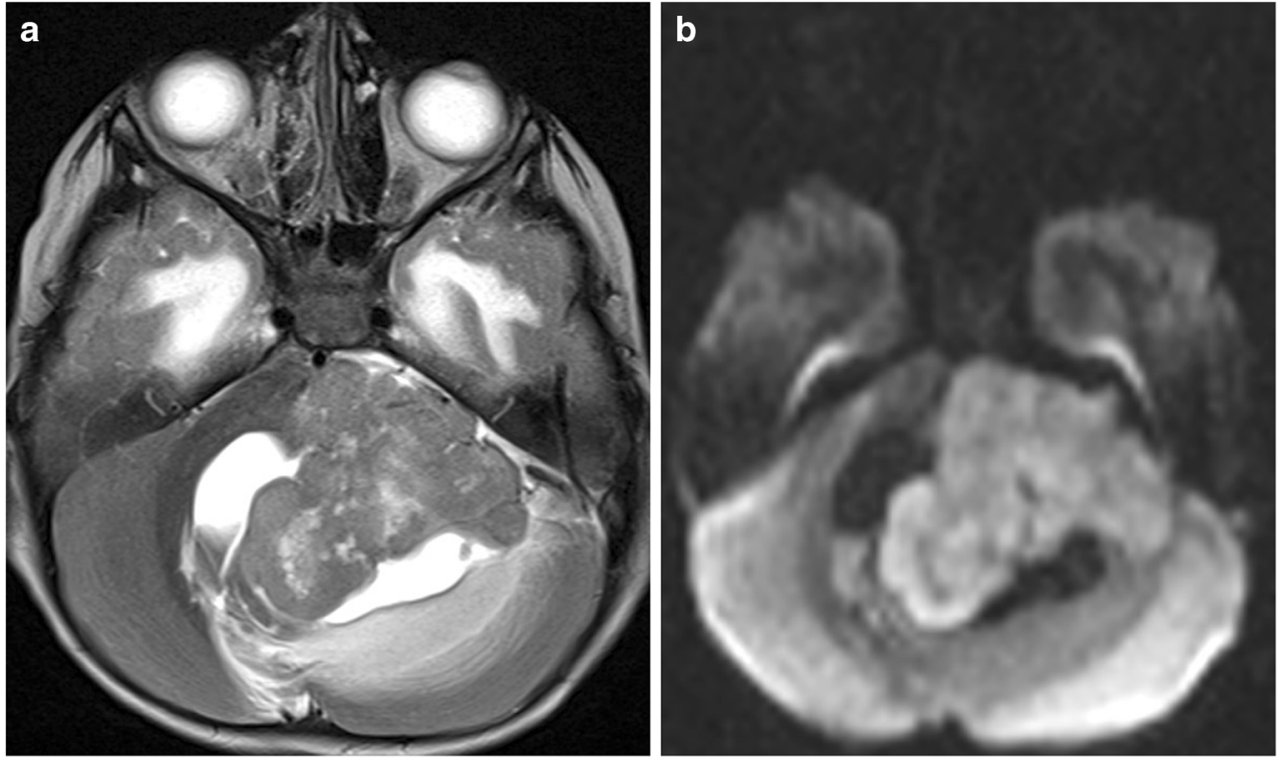

Pseudotumoral focal or diffuse enlargement of the brainstem (without neoplastic involvement) can be encountered in neurofibromatosis type I. There may be associated $\mathrm{T} 2$ hyperintense foci or hamartoma; these have a predilection for the brainstem, cerebellar peduncles and basal ganglia. Occasionally, patients may develop hydrocephalus secondary to a tectal hamartoma or a glioma causing secondary aqueductal stenosis (Fig. 28) [25, 26].

\section{Secondary involvement of paediatric brainstem}

It is crucial to remember that the brainstem can become secondarily involved because of direct extension of other CNS tumours especially from the posterior fossa region. Brainstem compression and/or infiltration by tumours such as medulloblastoma or gliomas is also not uncommon (Fig. 29) [24].
Extra-cranial solid tumour metastasising to the brain is a rarity in the paediatric age group though the brain can be a frequent site of involvement in haematological malignancies such as leukaemias. Brain involvement in leukaemia can be direct (due to leukaemic infiltration) (Fig. 30) or indirect. Indirect involvement occurs secondary to thrombosis or haemorrhage (due to hyperleucocytosis, sepsis and coagulopathy), or secondary to a neutropenic state predisposing the patient to infections (see Fig. 23), or as a sequela of chemotherapy-related encephalopathy or thrombotic microangiopathy [27].

\section{Conclusion}

A multifarious paediatric neurological disorders may primarily or secondarily involve the brainstem. Whilst these can be

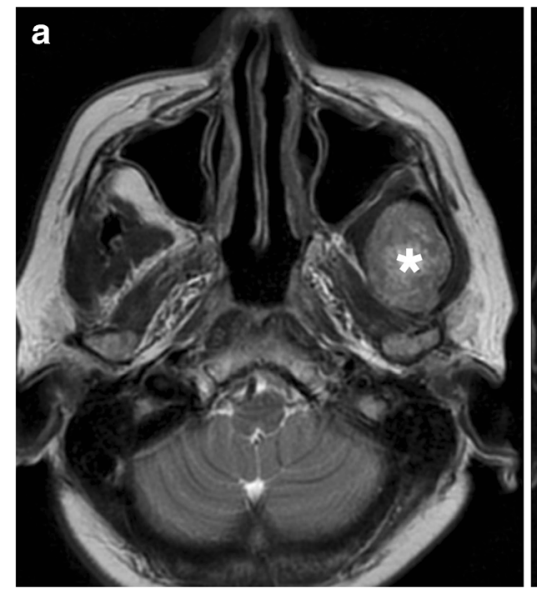

Fig. 30 A 17-year-old patient with acute myeloid leukaemia: a-b Axial $\mathrm{T} 2$ and post-contrast $\mathrm{T} 1$-weighted image shows an enhancing mass in the left masticator space suggestive of a chloroma (asterisk). c Axial T1-
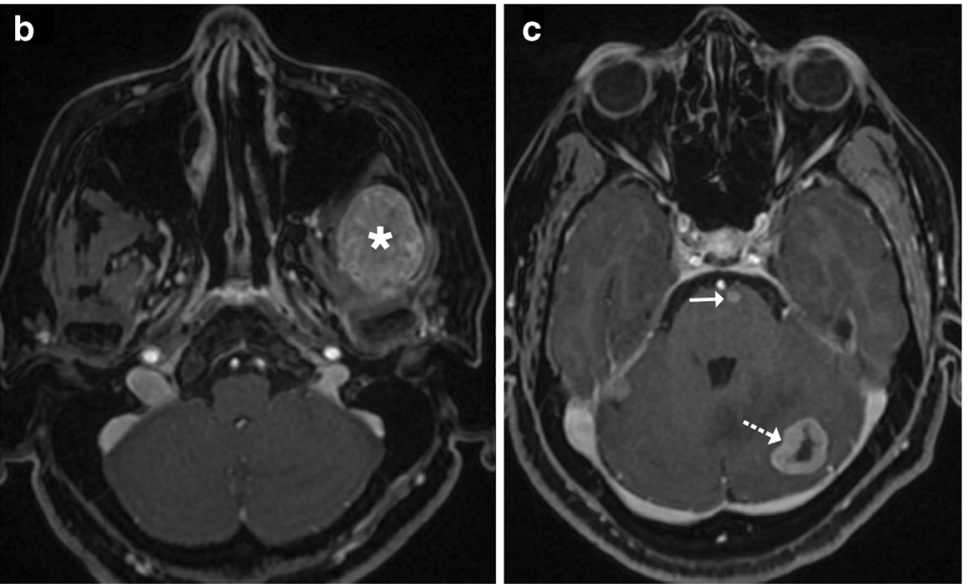

weighted image shows enhancing deposits in the left cerebellum (dotted arrow) and pons (arrow) 


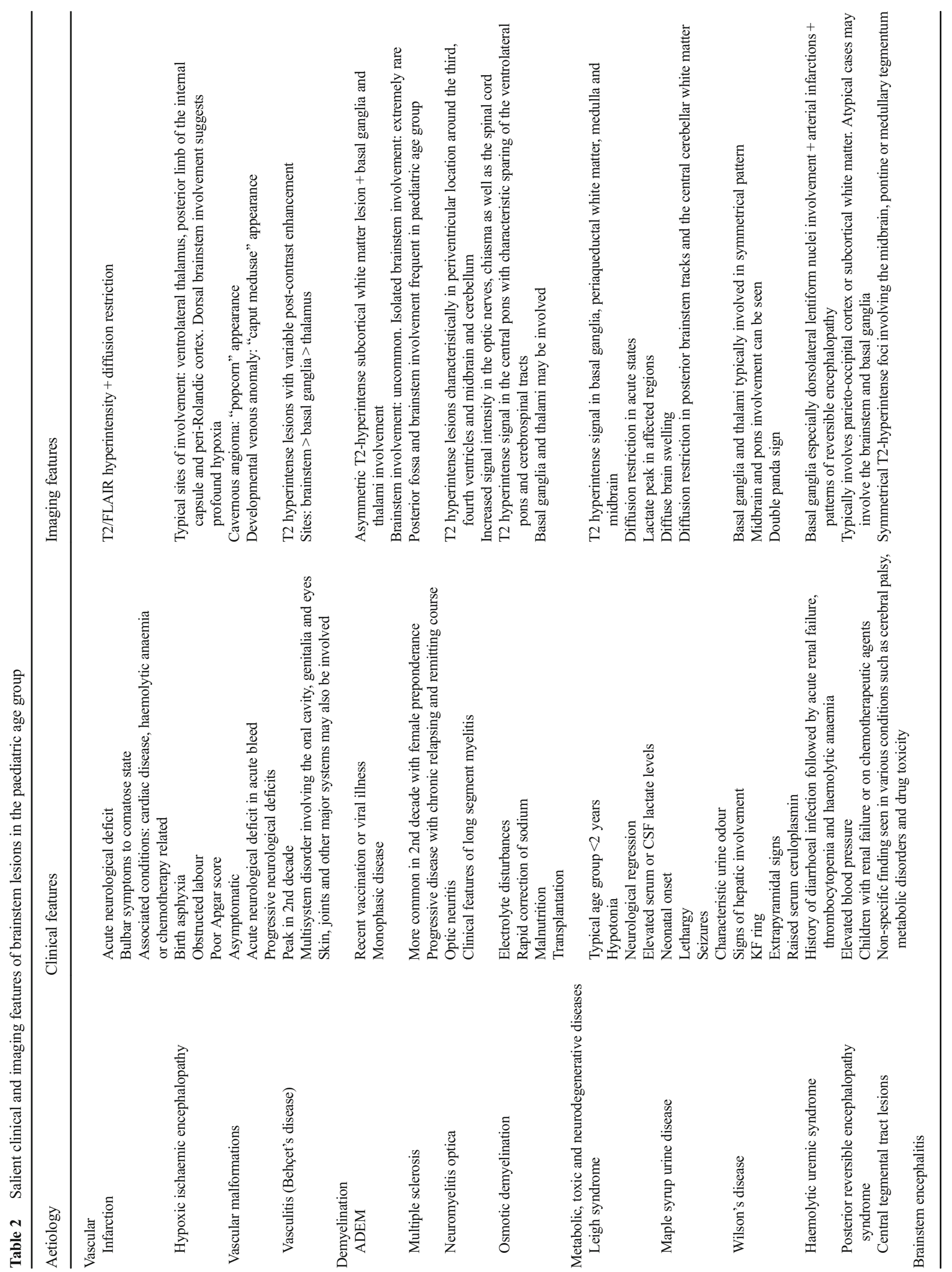


aetiologically classified as infections, metabolic and demyelinating disorders, vascular conditions, neurodegeneration and tumours, they often have overlapping clinical presentation. Moreover, their deep-seated nature and high risk of potential morbidity often precludes histopathological assessment. Thus their evaluation as well as follow-up primarily revolve around multi-planar MR imaging, which not only helps in localising the pathology but also aids in formulating a rational diagnosis. Whilst certain disorders such as Leigh syndrome and Maple syrup urine disease have certain characteristic imaging patterns, it is vital to understand that neither imaging nor clinical assessment alone may be able to clinch the diagnosis. Thus we as radiologists ought to be aware of the salient clinical features, laboratory parameters and radiological features that need to be looked into before we venture into reporting these pathologies. We hope that the comprehensive diagnostic approach (Table 2) outlined in this article will not only help the radiologists in acquainting themselves with the spectrum of paediatric brainstem pathologies, but also motivate them to work in close collaboration with their clinical counterparts, which would ensure improved clinical care offered to their paediatric patients.

Open Access This article is distributed under the terms of the Creative Commons Attribution 4.0 International License (http:// creativecommons.org/licenses/by/4.0/), which permits unrestricted use, distribution, and reproduction in any medium, provided you give appropriate credit to the original author(s) and the source, provide a link to the Creative Commons license, and indicate if changes were made.

\section{References}

1. Alper G, Sreedher G, Zuccoli G (2013) Isolated brain stem lesion in children: is it acute disseminated encephalomyelitis or not? AJNR Am J Neuroradiol 34:217-20

2. Jallo GI, Biser-Rohrbaugh A, Freed D (2004) Brainstem gliomas. Childs Nerv Syst 20:143-153

3. Pollack I, Hoffman HJ, Humphreys RP et al (1993) The long-term outcome after surgical treatment of dorsally exophytic brain-stem gliomas. J Neurosurg 78:859-63

4. Lanni G, Catalucci A, Conti L, Di Sibio A, Paonessa A, Gallucci M (2011) Pediatric stroke: clinical findings and radiological approach. Stroke Res Treat 2011:172168

5. Hegde AN, Mohan S, Lath N, Lim CC (2011) Differential diagnosis for bilateral abnormalities of the basal ganglia and thalamus. Radiographics 31:5-30

6. Huang BY, Castillo M (2008) Hypoxic-ischemic brain injury: imaging findings from birth to adulthood. Radiographics 28:417-39

7. Chae EJ, Do KH, Seo JB et al (2008) Radiologic and clinical findings of Behçet disease: comprehensive review of multisystemic involvement. Radiographics 28:e31

8. Hegde AN, Mohan S, Lim CC (2012) CNS cavernous haemangioma: "popcorn" in the brain and spinal cord. Clin Radiol 67:380-8

9. Vilanova JC, Barceló J, Smirniotopoulos JG, Pérez-Andrés R et al (2004) Hemangioma from head to toe: MR imaging with pathologic correlation. Radiographics 24:367-85 
10. Firat AK, Karakaş HM, Yakinci C, Altinok et al (2004) An unusual case of acute disseminated encephalomyelitis confined to brainstem. Magn Reson Imaging 22:1329-32

11. Ghassemi R, Antel SB, Narayanan S, Francis SJ et al (2008) Lesion distribution in children with clinically isolated syndromes. Ann Neurol 63:401-5

12. Verhey LH, Shroff M, Banwell B (2013) Pediatric multiple sclerosis: pathobiological, clinical, and magnetic resonance imaging features. Neuroimaging Clin N Am 23:227-43

13. Barnett Y, Sutton IJ, Ghadiri M, Masters L et al (2014) Conventional and advanced imaging in neuromyelitis optica. AJNR Am J Neuroradiol 35(8):1458-66

14. Kilicarslan R, Alkan A, Demirkol D, Toprak H et al (2012) Maple syrup urine disease: diffusion-weighted MRI findings during acute metabolic encephalopathic crisis. Jpn J Radiol 30:522-5

15. Singh P, Ahluwalia A, Saggar K et al (2011) Wilson's disease: MRI features. J Pediatr Neurosci 6:27-28

16. Steinborn M, Leiz S, Rüdisser K, Griebel M, Harder T et al (2004) $\mathrm{CT}$ and MRI in haemolytic uraemic syndrome with central nervous system involvement: distribution of lesions and prognostic value of imaging findings. Pediatr Radiol 34:805-10

17. Agarwal A, Kapur G, Altinok D (2015) Childhood posterior reversible encephalopathy syndrome: magnetic resonance imaging findings with emphasis on increased leptomeningeal FLAIR signal. Neuroradiol J 28:638-43

18. Yoshida S, Hayakawa K, Yamamoto A, Aida N et al (2009) Symmetrical centraltegmental tract (CTT) hyperintense lesions on magnetic resonance imaging in children. Eur Radiol 19:462-9
19. Co SJ, Mackenzie IR, Shewchuk JR (2015) Rabies encephalitis. RadioGraphics 35:235-238

20. Zagardo MT, Shanholtz CB, Zoarski GH, Rothman MI (1998) Rhombencephalitis caused by adenovirus: MR imaging appearance. AJNR Am J Neuroradiol 19:1901-1903

21. Sahaiu-Srivastava S, Jones B (2008) Brainstem tuberculoma in the immunocompetent: case report and literature review. Clin Neurol Neurosurg 110:302-4

22. Robert Y, Shih RY, Koeller KK (2015) Bacterial, fungal, and parasitic infections of the central nervous system: radiologic-pathologic correlation and historical perspectives. ${ }^{1}$. RadioGraphics 35:11411169

23. Rasalkar DD, Paunipagar BK, Sanghvi D, Sonawane BD et al (2011) Magnetic resonance imaging in cerebral malaria: a report of four cases. Br J Radiol 84:380-385

24. Barkovich AJ, Raybaud C (2012). Pediatric neuroimaging, 5th edn. Lippincott Williams \& Wilkins, Philadelphia

25. Ullrich NJ, Raja AI, Irons MB, Kieran MW, Goumnerova L (2007) Brainstem lesions in neurofibromatosis type 1 . Neurosurgery 61 : $762-6$

26. Dinçer A, Yener U, Özek MM (2011) Hydrocephalus in patients with neurofibromatosis type 1: MR imaging findings and the outcome of endoscopic third ventriculostomy. AJNR Am J Neuroradiol 32:643-6

27. Kembhavi SA, Somvanshi S, Banavali S, Kurkure P et al (2012) Pictorial essay: acute neurological complications in children with acute lymphoblastic leukemia. Indian J Radiol Imaging 22:98-105 